\title{
Dynamic Response of an Offshore Pile, a Poro-Elastic Seabed and Seawater due to Water Waves
}

\author{
Jian-Fei Lu* and Dong-Sheng Jeng ${ }^{\#}$
}

\begin{abstract}
*Department of Civil Engineering, JiangSu University, Zhenjiang, Jiangsu, 212013, P.R. China and ${ }^{\#}$ Division of Civil Engineering, School of Engineering, Physics and Mathematics, University of Dundee, Dundee, DD1 4HN, Scotland, UK
\end{abstract}

\begin{abstract}
In this study, a coupled model is developed to address the dynamic response of an offshore pile to linear water waves. In the coupled model, the pile and the seabed are treated as a saturated poro-elastic medium described by Biot's dynamic theory, while the seawater is considered as a conventional acoustic medium. To establish the coupled model, boundary element formulations for saturated porous media are derived for the pile and the seabed, respectively, and the acoustic boundary element formulation is constructed for the seawater. The coupled boundary element model is obtained using these three boundary element formulations as well as the continuity conditions along the interfaces between the pile, the seabed and the seawater. In the model, linear water waves are considered as an external load and its load is evaluated via the wave function expansion method. Numerical results shows that increment of the modulus ratio between the pile and the seabed can reduce the horizontal displacement of the pile and the pore pressure of the seabed around the pile. Also, the maximum pore pressure of the seabed usually occurs at the upper part of the seabed around the pile.
\end{abstract}

\section{INTRODUCTION}

It is well-known that sea waves can cause enormous loads on the offshore structures, particularly, in rough weather conditions. Thus, motivated by the need of safe recovery of oil and natural gas in the offshore region, ocean engineers have paid much attenuation to the determination of the wave loads on offshore structures. However, due to incomplete knowledge concerning offshore structures as well as their environmental factors and resulting inappropriate designs, some marine structure failure incidents have occurred recent years [1-3]. Therefore, a safe design of an offshore structure remains a challenge for ocean engineers.

The pile foundation is a very common and important structure for offshore engineering, which can be used as the foundation for offshore wind farms, offshore platforms, offshore ports, long-span cross-bay bridges etc. To date, numerous researches concerning offshore piles and water waves have been carried out. For example, the effects of various waves on offshore piles have been investigated by many investigations [4-6]. The offshore pile capacity and stresses and strain of a pile due to wave loads were addressed in [7, 8], respectively. Mitwally and Novak [9] used a linear analysis to address dynamic interaction between pile-soil-pile, when the system is subjected to a random wave loading. Moreover, using the concept of dynamic $\mathrm{p}-\mathrm{y}$ curves, the response of fixed offshore platforms to wave and current loading when taking into account the soil-pile interaction was investigated by Mostafa \& Naggar [10].

It is worthwhile stressing that existing researches about offshore piles, the seabed and the seawater mainly focused

\footnotetext{
*Address correspondence to this author at the Department of Civil Engineering, JiangSu University, Zhenjiang, Jiangsu, 212013, P.R. China; Email: 1jfdoctor@yahoo.com and Division of Civil Engineering, School of Engineering, Physics and Mathematics, University of Dundee, Dundee, DD1 4HN, Scotland, UK; Email: d.jeng@dundee.ac.uk
}

on separate aspects of the problem, rather than treated them as a coupled system. However, in natural ocean environments, the pile, the seabed and the seawater are coupled. Therefore, response of the pile is related to those of the seabed and the seawater. Furthermore, besides the design for the pile itself, a complete design for offshore pile foundation should also include the design for the seabed, which aims to guarantee the seabed have enough strength to resist liquefaction and shear failures. Obviously, the design of the seabed will unavoidably involve detailed knowledge concerning the stresses, the deformation and the pore pressure of the seabed, which can only be available via a coupled analysis of the pile-seabed-seawater system. Consequently, a coupled model for the offshore pile, the seabed and the seawater is crucial for a successful design for the offshore pile foundation and its surrounding seabed. Another limitation of existing researches concerning the pile-seabed interaction is that only single phase medium model is used to describe the seabed. Nevertheless, it is well-known that the seabed is a porous medium saturated by seawater and more importantly, pore fluid of the seabed plays a very important role in the liquefaction and the shear failure of the seabed. Thus, an appropriate coupled model for the pile-seabed-seawater system should be able to account for the role of the pore fluid of the seabed. In this study, a coupled model is developed to investigate the interaction between the pile, the seabed and the seawater, when the system is subjected to linear water waves. The main contributions of this paper are as follows. First, we propose the idea to treat the pile, the seabed, the seawater as a whole system, which has not appeared in the literature to date. Secondly, the coupled model for the pile, the poro-elastic seabed and the seawater is established technically for the first time by combining the BEM formulations for the pile, the seabed and seawater via the continuity conditions along their interfaces. Also, the treatment of the Cauchy type integral for the half space seawater belongs to our own contribution. 
The remainder of this paper is organized as follows. First, Airy linear wave theory [11] is used to calculate the external load applied to the system due to linear water waves. Second, Biot's theory $[12,13]$ is outlined. Then, the boundary element formulations for the poroelastic medium and the acoustic medium are established. Three BEM formulations for the pile, the seabed and the seawater are coupled together to form the coupled BEM model for the system. Numerical examples are presented to demonstrate the capacity of the proposed model.

\section{EXTERNAL LOADS APPLIED TO THE SYSTEM DUE TO LINEAR WATER WAVES}

In this study, linear water waves are considered as the external load applied to the pile and the seabed. When evaluating the external load due to linear water waves, the pile and the seabed are assumed to be fixed. The external load obtained in this section will be applied to the pile-seabedseawater system to calculate the response of the system via the coupled model.

As only frequency domain response of the pile-seabedseawater system is considered in this paper, as a result, only the monochromatic linear water wave or the frequency domain linear water wave is involved here. The waves which have contribution to the external load applied to the pile and the seabed consist of two parts: the incident wave and the scattered wave. Thus, the velocity potential for the total external load has the following expression,

$$
\hat{\phi}=\hat{\phi}^{(I)}+\hat{\phi}^{(S)}
$$

where $\hat{\phi}^{(I)}$ and $\hat{\phi}^{(S)}$ are the velocity potentials for the incident wave and the scattered wave, respectively and a caret above a variable denotes the frequency domain. The velocity potential for the incident plane wave is given by

$$
\hat{\phi}^{(I)}=A \times \operatorname{ch}\left[k\left(z-h_{2}\right)\right] e^{i(\omega t-k y)}=G(z, t) e^{-i k y} .
$$

where $h_{2}$ is the depth of the seawater, and $A$ is determined by the expression

$$
A=\frac{g H}{2 i \omega \cosh k h_{2}}
$$

where $H$ is the wave height of the incident wave and $g$ is the gravitational acceleration. The angular frequency $\omega$ and the wave-number of the incident wave fulfill the following dispersion relation

$$
\omega^{2}=-g k t h\left(-k h_{2}\right)
$$

It is noted that downward direction of $z$-axis is assumed to be positive, as depicted in Fig. (1). To determine the scattered wave field analytically, it is appropriate to study the current problem in the cylindrical coordinate system as shown in Fig. (1). Thus, the incident wave given by (2) can be expanded into the following series expression [11]

$$
\begin{aligned}
\hat{\phi}^{(I)}= & G(z, t) e^{-i k y}=G(z, t)\left[J_{0}(k r)\right. \\
& \left.+\sum_{n=1}^{\infty} 2(-i)^{n} J_{n}(k r) \cos (n \theta)\right]
\end{aligned}
$$

In the cylindrical coordinate system (Fig. (1)), the scattered wave field has the following series expression

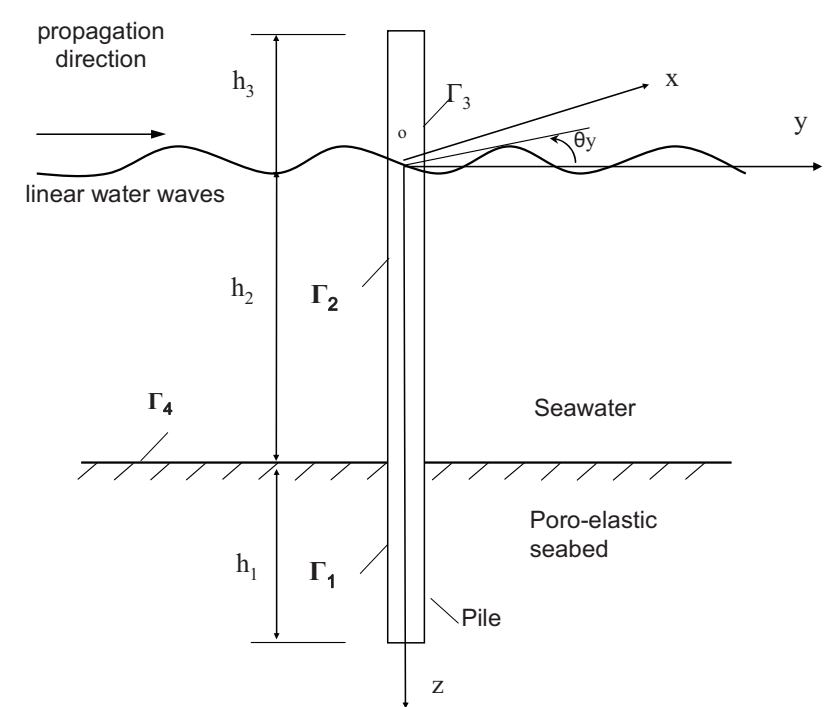

Fig. (1). An offshore pile, the seabed and the seawater subjected to linear water waves.

$$
\begin{aligned}
\hat{\phi}^{(S)} & =G(z, t)\left\{A_{0} H_{0}^{(2)}(k r)\right. \\
& \left.+\sum_{n=1}^{\infty}\left[A_{n} H_{n}^{(2)}(k r) \cos (n \theta)+B_{n} H_{n}^{(2)}(k r) \sin (n \theta)\right]\right\}
\end{aligned}
$$

Since the incident wave and the scattered wave field given in (5) and (6) already satisfy the boundary conditions at the water surface and the surface of the seabed, thus, only the boundary condition along the pile boundary confines the solution for the incident wave and the scattered wave. The boundary condition along the pile boundary reads

$$
\left.\frac{\partial\left(\hat{\phi}^{(I)}+\hat{\phi}^{(S)}\right)}{\partial r}\right|_{r=a}=0
$$

where $a$ is the radius of the pile. Substitution of equations (5) and (6) into equation (7) yields the expressions for the coefficients $A_{0}, A_{n}$ and $B_{n}$ in equation (6)

$$
\begin{gathered}
A_{0}=-\frac{J_{0}{ }^{\prime}(k a)}{H_{0}^{(2)}(k a)}, \quad A_{n}=-\frac{2(-i)^{n} J_{n}^{\prime}(k a)}{H_{n}^{(2)^{\prime}}(k a)}, \\
B_{n}=0, \quad n=1 \sim \infty
\end{gathered}
$$

The pressure due to the incident and the scattered wave has the following expression

$$
\hat{p}_{w}=\rho_{w} g z-\rho_{w} \frac{\partial\left(\hat{\phi}^{(I)}+\hat{\phi}^{(S)}\right)}{\partial t}
$$

where $\rho_{w}$ is the density of the seawater. Note that equations (5)-(6) and (8)-(9) determine the pressure exerting on the pile boundary and the seabed surface.

\section{BIOT'S PORO-ELASTIC THEORY}

The constitutive equations for a homogeneous porous medium can be expressed as [13]

$$
\begin{aligned}
& \sigma_{i j}=2 \mu \varepsilon_{i j}+\lambda \delta_{i j} e-\alpha \delta_{i j} p \\
& p=-\alpha M e+M \zeta \\
& e=u_{i, i}, \zeta=-w_{i, i}
\end{aligned}
$$


in which $u_{i}$ and $w_{i}$ denote the average solid displacement and the infiltration displacement of the pore fluid; $\varepsilon_{i j}$ and $e$ are the strain tensor and the dilatation of the solid skeleton; $\zeta$ is the volume of fluid injected into a unit volume of the bulk material; $\sigma_{i j}$ is the stress of the bulk porous medium; $p$ is the excess pore pressure and $\delta_{i j}$ is the Kronecker delta. The compressibility of the saturated porous medium is considered in terms of the solid skeleton Lamè constants, $\lambda$ and $\mu$, and Biot's parameters $\alpha$ and $M$ [14]

The bulk density of a porous medium with the solid skeleton of density $\rho_{s}$, the pore fluid of density $\rho_{f}$, and porosity $\phi$, can be expressed as $\rho_{b}=(1-\varphi) \rho_{s}+\varphi \rho_{f}$. Using a superimposed dot to denote the time derivative, the equations of motion for the bulk porous medium and the pore fluid are [13]

$$
\begin{gathered}
\mu u_{i, j j}+\left(\lambda+\alpha^{2} M+\mu\right) u_{j, j i} \\
+\alpha M w_{j, j i}+F_{i}=\rho_{b} \ddot{u}_{i}+\rho_{f} \ddot{w}_{i} \\
\alpha M u_{j, j i}+M w_{j, j i}+f_{i}=\rho_{f} \ddot{u}_{i} \\
+m \ddot{w}_{i}+\frac{\eta}{k} \dot{w}_{i}
\end{gathered}
$$

where $\eta$ and $k$ are the viscosity of the pore fluid and the permeability of the porous medium respectively; $F_{i}$ and $f_{i}$ are the body forces applied to the porous medium and the pore fluid; and $m=a_{\infty} \rho_{f} / \varphi$, in which $a_{\infty}$ is tortuosity.

In this study, to investigate the frequency domain response of the pile, the seabed and the seawater, the Fourier transform with respect to time and frequency is applied and defined as follows [15]

$$
\begin{aligned}
& \hat{f}(\omega)=\int_{-\infty}^{+\infty} f(t) e^{-i \omega t} d t \\
& f(t)=\frac{1}{2 \pi} \int_{-\infty}^{+\infty} \hat{f}(\omega) e^{i \omega t} d \omega
\end{aligned}
$$

where $t$ and $\omega$ denote time and frequency, respectively; and a caret above the variable denotes the Fourier transform.

According to the analysis of Bonnet [16], although two displacement vectors are used in Biot's theory, there are only four independent variables in the two-phase porous medium. Therefore, Biot's theory is reformulated in terms of four independent variables (three displacement components for the solid skeleton and the pore fluid pressure) as follows

$$
\begin{gathered}
\mu \hat{u}_{i, j j}+(\lambda+\mu) \hat{u}_{j, j i}+\rho_{g} \omega^{2} \hat{u}_{i} \\
-\alpha_{g} \hat{p}_{, i}=-\hat{F}_{g i} \\
\hat{p}_{, j j}+\beta_{2} \omega^{2} \hat{p}-\beta_{3} \hat{u}_{j, j}=-\hat{\vartheta}_{f}
\end{gathered}
$$

where the constants in (14) and (15) have the following expressions

$$
\begin{aligned}
& \beta_{1}=M /\left[m \omega^{2}-i \omega(\eta / k)\right] \\
& \beta_{2}=1 /\left(\beta_{1} \omega^{2}\right) \\
& \beta_{3}=\rho_{f} \omega^{2}-\alpha\left[m \omega^{2}-i \omega(\eta / k)\right] \\
& \beta_{4}=\rho_{f} \omega^{2} \beta_{1} / M \\
& \alpha_{g}=\alpha-\beta_{4} \\
& \rho_{g}=\rho_{b}-\beta_{4} \rho_{f} \\
& \hat{F}_{g i}=\hat{F}_{i}-\beta_{4} \hat{f}_{i} \\
& \hat{\vartheta}_{f}=-\hat{f}_{j, j}
\end{aligned}
$$

\section{BOUNDARY ELEMENT METHOD FOR A POROUS MEDIUM AND SEAWATER}

In this study, the pile and the seabed are treated as poroelastic medium, and the seawater is considered as a conventional acoustic medium. In this section, the boundary element method for the poro-elastic medium will be outlined. Also, the boundary element method for the acoustic medium with an infinite free surface will be detailed. The treatment of the Cauchy-type singularity involved in the boundary integral equation for the acoustic medium will also be discussed in this section.

\section{Boundary Element Method (BEM) For The Porous Medium}

Boundary element method (BEM) for the porous medium has been addressed by many researches. For example, a complete boundary integral equation for saturated porous media was formulated by Dominguez $[17,18]$ using three solid displacements and a fourth variable proportional to the pore pressure. By considering the same fundamental variables, Cheng et al. [19] developed a boundary integral equation for porous media in the frequency domain using a 3-D boundary integral equation. The $3-\mathrm{D}$ problem of wave scattering within a porous medium in the frequency domain was later addressed by Zimmerman and Stern [20]. In this study, based on the reciprocal work theorem, the boundary integral equation for the porous medium is formulated in terms of three solid displacements and the pore pressure.

To formulate the boundary integral equation for the porous medium, Green's function for the porous medium is required in advance. Green's function of the porous medium consists of two parts: one part is due to a point force applied to the solid skeleton and the other part is due to a dilatation source applied within the pore fluid.

For a 3-D Green's function due to the point force applied to the solid skeleton, Green's function $\hat{\mathbf{U}}^{G_{s}}$ and $\hat{\mathbf{P}}^{G_{s}}$ can be represented by three scalar potentials as follows [20,21]

$$
\begin{aligned}
\hat{\mathbf{U}}^{G_{s}}=\nabla \nabla \hat{\phi}_{f}^{G_{s}}+\nabla \nabla \hat{\phi}_{s}^{G_{s}} \\
+\left(\nabla^{2} \hat{\psi}^{G_{s}} \mathbf{I}_{3 \times 3}-\nabla \nabla \hat{\psi}^{G_{s}}\right)
\end{aligned}
$$




$$
\hat{\mathbf{P}}^{G_{s}}=A_{f} \nabla \nabla^{2} \hat{\phi}_{f}^{G_{s}}+A_{s} \nabla \nabla^{2} \hat{\phi}_{s}^{G_{s}}
$$

in which the constants $A_{f}, A_{s}$ are available in [22]. The potentials, $\hat{\phi}_{f}^{G_{s}}, \hat{\phi}_{s}^{G_{s}}$ and $\hat{\psi}^{G_{s}}$ in (24)-(25) are given as follows

$$
\begin{aligned}
\hat{\phi}_{f}^{G_{s}} & =\frac{d_{s}}{d_{s}-d_{f}} \frac{1}{4 \pi \rho_{g} \omega^{2} r}\left[1-e^{-i k_{f} r}\right] \\
\hat{\phi}_{s}^{G_{s}} & =\frac{d_{f}}{d_{f}-d_{s}} \frac{1}{4 \pi \rho_{g} \omega^{2} r}\left[1-e^{-i k_{s} r}\right] \\
\hat{\psi}^{G_{s}} & =\frac{1}{4 \pi \mu k_{t}^{2} r}\left[1-e^{-i k_{t} r}\right]
\end{aligned}
$$

where

$$
\begin{aligned}
& r=\sqrt{x_{k} x_{k}} \\
& d_{f}=\beta_{2} A_{f} \omega^{2}-\beta_{3} \\
& d_{s}=\beta_{2} A_{s} \omega^{2}-\beta_{3}
\end{aligned}
$$

In (26)(28), $k_{f}, k_{s}, k_{t}$ are the complex wave-number for the P1, P2 and the shear wave of the porous medium [22].

For the Green's function due to a dilatation source applied within the pore fluid, Green's function $\hat{\mathbf{u}}^{G_{f}}$ and $\hat{p}^{G_{f}}$ can be represented by two scalar potentials and one vector potential as follows

$$
\begin{aligned}
& \hat{\mathbf{u}}^{G_{f}}=\nabla \hat{\phi}_{f}^{G_{f}}+\nabla \hat{\phi}_{s}^{G_{f}}+\nabla \times \nabla \times \hat{\Psi}^{G_{f}} \\
& \hat{p}^{G_{f}}=A_{f} \nabla^{2} \hat{\phi}_{f}^{G_{f}}+A_{s} \nabla^{2} \hat{\phi}_{s}^{G_{f}}
\end{aligned}
$$

where the potentials $\hat{\phi}_{f}^{G_{f}}, \hat{\phi}_{s}^{G_{f}}$ and $\hat{\Psi}^{G_{f}}$ have the form

$$
\begin{aligned}
& \hat{\phi}_{f}^{G_{f}}=\frac{1}{4 \pi\left(d_{f}-d_{s}\right) r}\left[1-e^{-i k_{f} r}\right] \\
& \hat{\phi}_{s}^{G_{f}}=\frac{1}{4 \pi\left(d_{s}-d_{f}\right) r}\left[1-e^{-i k_{s} r}\right] \\
& \hat{\Psi}^{G_{f}}=0
\end{aligned}
$$

The reciprocal work theorem for the porous medium has the following form [19]

$$
\hat{\sigma}_{i j}^{(1)} \hat{\varepsilon}_{i j}^{(2)}+\hat{p}^{(1)} \hat{\zeta}^{(2)}=\hat{\sigma}_{i j}^{(2)} \hat{\varepsilon}_{i j}^{(1)}+\hat{p}^{(2)} \hat{\zeta}^{(1)}
$$

where two states are represented by superscripts 1 and 2, respectively.

Integration of equation (37) over an arbitrary domain $\Omega$ gives the reciprocal work theorem for the porous medium as

$$
\begin{aligned}
& \int_{\Omega}\left(\hat{\sigma}_{i j}^{(1)} \hat{\varepsilon}_{i j}^{(2)}+\hat{p}^{(1)} \hat{\zeta}^{(2)}\right) d \Omega= \\
& \int_{\Omega}\left(\hat{\sigma}_{i j}^{(2)} \hat{\varepsilon}_{i j}^{(1)}+\hat{p}^{(2)} \hat{\xi}^{(1)}\right) d \Omega
\end{aligned}
$$

Using (38) and the governing equations for the porous medium, the generalised Betti's formula for the porous medium has the form

$$
\begin{aligned}
& \int_{S}\left[\hat{t}_{j}^{(1)} \hat{u}_{j}^{(2)}-\hat{t}_{j}^{(2)} \hat{u}_{j}^{(1)}\right] d S-\int_{S}\left[\hat{p}^{(1)} \hat{w}_{n}^{(2)}-\hat{p}^{(2)} \hat{w}_{n}^{(1)}\right] d S= \\
& \int_{\Omega}\left[\hat{p}^{(1)} \hat{V}_{f}^{(2)}-\hat{p}^{(2)} \hat{V}_{f}^{(1)}\right] d \Omega-\int_{\Omega}\left[\hat{F}_{g j}^{(1)} \hat{u}_{j}^{(2)}-\hat{F}_{g j}^{(2)} \hat{u}_{j}^{(1)}\right] d \Omega
\end{aligned}
$$

where

$$
\begin{aligned}
& \hat{t}_{j}^{(\chi)}=\hat{\sigma}_{j k}^{(\chi)} n_{k}, \quad \chi=1,2 \\
& \hat{w}_{n}^{(\chi)}=\left(\beta_{1} / M\right)\left[\hat{p}_{j}^{(\chi)}-\rho_{f} \omega^{2} \hat{u}_{j}^{(\chi)}\right] n_{j}, \quad \chi=1,2 \\
& \hat{V}_{f}^{(\chi)}=\left(\beta_{1} / M\right) \hat{\vartheta}_{f}^{(\chi)}, \quad \chi=1,2
\end{aligned}
$$

To derive the Somigliana identity for the porous medium, state 1 is now defined as the real state, assuming that there are no body forces acting on the solid skeleton and the pore fluid, while state 2 corresponds to the Green's function of the porous medium. Thus, the displacement of the solid frame is obtained by considering a unit point force acting in the $i$-direction at a point $\mathbf{x}$

$$
\begin{aligned}
& \hat{u}_{i}(\mathbf{x}, \omega)= \\
& \int_{S}\left[\hat{U}_{i j}^{G_{s}}(\mathbf{x}, \boldsymbol{\xi}, \omega) \hat{t}_{j}(\boldsymbol{\xi}, \omega)-\hat{T}_{i j}^{G_{s}}(\mathbf{x}, \boldsymbol{\xi}, \omega) \hat{u}_{j}(\boldsymbol{\xi}, \omega)\right] d S(\boldsymbol{\xi}) \\
& \quad-\int_{S}\left[\hat{W}_{n i}^{G_{s}}(\mathbf{x}, \boldsymbol{\xi}) \hat{p}(\boldsymbol{\xi})-\hat{P}_{i}^{G_{s}}(\mathbf{x}, \boldsymbol{\xi}) \hat{w}_{n}(\boldsymbol{\xi})\right] d S(\boldsymbol{\xi})
\end{aligned}
$$

where the Green's function $\hat{T}_{i j}^{G_{s}}(\mathbf{x}, \boldsymbol{\xi})$ and $\hat{W}_{n i}^{G_{s}}(\mathbf{x}, \boldsymbol{\xi})$ are given by

$$
\begin{gathered}
\hat{\sigma}_{i j k}^{G_{s}}=\mu\left[\hat{U}_{i j, k}^{G_{s}}+\hat{U}_{i k, j}^{G_{s}}\right]+\lambda \hat{U}_{i l, l}^{G_{s}} \delta_{j k}, \\
-\alpha \hat{P}_{i}^{G_{s}} \delta_{j k} \\
\hat{T}_{i j}^{G_{s}}(\mathbf{x}, \boldsymbol{\xi}, \omega)=\hat{\sigma}_{i j k}^{G_{s}}(\mathbf{x}, \boldsymbol{\xi}, \omega) n_{k}(\boldsymbol{\xi}) \\
=\mu\left[\hat{U}_{i j, k}^{G_{s}}(\mathbf{x}, \boldsymbol{\xi}, \omega)+\hat{U}_{i k, j}^{G_{s}}(\mathbf{x}, \boldsymbol{\xi}, \omega)\right] n_{k}(\boldsymbol{\xi}) \\
+\lambda \hat{U}_{i l, l}^{G_{s}}(\mathbf{x}, \boldsymbol{\xi}, \omega) n_{j}(\boldsymbol{\xi})-\alpha \hat{P}_{i}^{G_{s}}(\mathbf{x}, \boldsymbol{\xi}, \omega) n_{j}(\boldsymbol{\xi}) \\
\hat{W}_{n i}^{G_{s}}(\mathbf{x}, \boldsymbol{\xi}, \omega)=\frac{\beta_{1}}{M}\left[\frac{\partial \hat{P}_{i}^{G_{s}}(\mathbf{x}, \boldsymbol{\xi}, \omega)}{\partial \xi}\right. \\
\left.-\rho_{f} \omega^{2} \hat{U}_{i j}^{G_{s}}(\mathbf{x}, \boldsymbol{\xi}, \omega)\right] n_{j}(\boldsymbol{\xi})
\end{gathered}
$$

Similarly, considering a dilatation source acting at a point $\mathbf{X}$ within the pore fluid gives 


$$
\begin{aligned}
\hat{p}(\mathbf{x}, \omega)= & \int_{S} \hat{U}_{j}^{G_{f}^{\prime}}(\mathbf{x}, \boldsymbol{\xi}, \omega) \hat{t}_{j}(\boldsymbol{\xi}, \omega) \\
& \left.-\hat{T}_{j}^{G_{f}^{\prime}}(\mathbf{x}, \boldsymbol{\xi}, \omega) \hat{u}_{j}(\boldsymbol{\xi}, \omega)\right] d S(\boldsymbol{\xi}) \\
-\int_{S} & {\left[\hat{W}_{n}^{G_{f}^{\prime}}(\mathbf{x}, \boldsymbol{\xi}, \omega) \hat{p}(\boldsymbol{\xi}, \omega)\right.} \\
& \left.-\hat{P}^{G_{f}^{\prime}}(\mathbf{x}, \boldsymbol{\xi}, \omega) \hat{w}_{n}(\boldsymbol{\xi}, \omega)\right] d S(\boldsymbol{\xi})
\end{aligned}
$$

where $\hat{T}_{j}^{G_{f}^{\prime}}$ and $\hat{W}_{n}^{G_{f}^{\prime}}$ have similar expressions as (44)-(46). It is noted that the Green's function $\hat{U}_{j}^{G_{f}^{\prime}}, \hat{T}_{j}^{G_{f}^{\prime}}, \hat{W}_{n}^{G_{f}^{\prime}}$ and $\hat{P}^{G_{f}^{\prime}}$ in (47) is different from the 3-D Green's function due to the dilatation source given by (32)-(36), the relations between the two being

$$
\begin{aligned}
& \hat{U}_{j}^{G_{f}^{\prime}}=\frac{M}{\beta_{1}} \hat{U}_{j}^{G_{f}}, \hat{T}_{j}^{G_{f}^{\prime}}=\frac{M}{\beta_{1}} \hat{T}_{j}^{G_{f}} \\
& \hat{W}_{n}^{G_{f}^{\prime}}=\frac{M}{\beta_{1}} \hat{W}_{n}^{G_{f}}, \hat{P}^{G_{f}^{\prime}}=\frac{M}{\beta_{1}} \hat{P}^{G_{f}}
\end{aligned}
$$

Introducing the following pseudo-displacement and pseudo-traction vectors and Green's functions

$$
\begin{aligned}
& \hat{\mathbf{u}}=\left[\begin{array}{llll}
\hat{u}_{1} & \hat{u}_{2} & \hat{u}_{3} & \hat{u}_{4}
\end{array}\right]^{T}=\left[\begin{array}{llll}
\hat{u}_{1} & \hat{u}_{2} & \hat{u}_{3} & \hat{p}
\end{array}\right]^{T} \\
& \hat{\mathbf{t}}=\left[\begin{array}{llll}
\hat{t}_{1} & \hat{t}_{2} & \hat{t}_{3} & \hat{t}_{4}
\end{array}\right]^{T}=\left[\begin{array}{llll}
\hat{t}_{1} & \hat{t}_{2} & \hat{t}_{3} & \hat{w}_{n}
\end{array}\right]^{T} \\
& \hat{\mathbf{U}}^{G^{*}}=\left[\begin{array}{ll}
\hat{U}_{m n}^{G^{*}}
\end{array}\right]=\left[\begin{array}{ll}
\hat{U}_{i j}^{G_{s}} & \hat{P}_{i}^{G_{s}} \\
\hat{U}_{j}^{G_{f}^{\prime}} & \hat{P}^{G_{f}^{\prime}}
\end{array}\right] \\
& \hat{\mathbf{T}}^{G^{*}}=\left[\begin{array}{ll}
\hat{T}_{m n}^{G}
\end{array}\right]=\left[\begin{array}{ll}
\hat{T}_{i j}^{G_{s}} & \hat{W}_{n i}^{G_{s}} \\
\hat{T}_{j}^{G_{f}^{\prime}} & \hat{W}_{n}^{G_{f}^{\prime}}
\end{array}\right], m, n=1,2,3,4
\end{aligned}
$$

and combining equations (43) and (47) yields

$$
\begin{array}{r}
\hat{u}_{i}(\mathbf{x}, \omega)=\int_{S} \hat{U}_{i j}^{G^{*}}(\mathbf{x}, \boldsymbol{\xi}, \omega) \hat{t}_{j}(\boldsymbol{\xi}, \omega) d S(\boldsymbol{\xi}) \\
-\int_{S} \hat{T}_{i j}^{G^{*}}(\mathbf{x}, \boldsymbol{\xi}, \omega) \hat{u}_{j}(\boldsymbol{\xi}, \omega) d S(\boldsymbol{\xi}) \\
i, j=1,2,3,4
\end{array}
$$

Performing a limit process as outlined by Zimmerman \& Stern [20], the following boundary integral equation for the porous medium can be obtained from equation (50)

$$
\begin{array}{r}
c_{i j}(\mathbf{x}) \hat{u}_{j}(\mathbf{x}, \omega)=\int_{S} \hat{U}_{i j}^{G^{*}}(\mathbf{x}, \boldsymbol{\xi}, \omega) \hat{t}_{j}(\xi, \omega) d S(\xi) \\
-\int_{S} \hat{T}_{i j}^{G^{*}}(\mathbf{x}, \boldsymbol{\xi}, \omega) \hat{u}_{j}(\boldsymbol{\xi}, \omega) d S(\xi) \\
i, j=1,2,3,4
\end{array}
$$

in which the free term $c_{i j}$ depends on the local boundary geometry around the point $\mathbf{x}$, with $c_{i j}=1 / 2 \delta_{i j}$ for $\mathbf{x}$ be- longing to a smooth part of the boundary. The second integral in (51) involving $\hat{T}_{i j}^{G^{*}}(\mathbf{x}, \boldsymbol{\xi}, \omega)$ should be understood in the sense of the Cauchy principal value. The Cauchy principal value integrals in (51) needs special treatment to be evaluated numerically. In this study, we use the auxiliary problem method proposed by Zimmerman \& Stern [20] to treat the Cauchy principal value integrals in (51). Thus, direct evaluation of the Cauchy principal value integrals in (51) is avoided.

\section{Boundary Element Method (BEM) For The Seawater}

As mentioned previously, the seawater here is considered as an acoustic medium, the pressure of which is described by the following Helmholtz equation

$$
\nabla^{2} \hat{p}_{w}+k_{w}^{2} \hat{p}_{w}=0
$$

The displacement of the seawater is given by the following equation

$$
\hat{\mathbf{q}}=\frac{1}{\rho_{w} \omega^{2}} \nabla \hat{p}_{w}
$$

For the seawater above the seabed, due to the existence of the infinite free surface of the water layer, thus, to save computational time, a boundary integral equation which can avoid the discretization of the free surface is preferable. Consequently, half space boundary integral equation and Green's function $[23,24]$ are used in this research

$$
c \hat{p}_{w}(\mathbf{x})=\int_{\Gamma}\left[\hat{p}_{w}^{G_{w}}(\mathbf{x}, \boldsymbol{\xi}) \hat{q}_{n}(\boldsymbol{\xi})-\hat{q}_{n}^{G_{w}}(\mathbf{x}, \boldsymbol{\xi}) \hat{p}_{w}(\boldsymbol{\xi})\right] d \Gamma(\boldsymbol{\xi})
$$

where $\hat{p}_{w}^{G_{w}}$ and $\hat{q}_{n}^{G_{w}}$ are the Green's function for the half space seawater, $c$ depends upon the local geometry at the point $\mathbf{x}$ and $\hat{q}_{n}(\boldsymbol{\xi})=\left[1 /\left(\rho_{w} \omega^{2}\right)\right] \partial \hat{p}_{w}(\boldsymbol{\xi}) / \partial \mathbf{n}(\boldsymbol{\xi}), \mathbf{n}(\boldsymbol{\xi})$ is the outward normal for the boundary $\Gamma$. The Green's function involved in (54) is given by

$$
\begin{aligned}
& \hat{p}_{w}^{G_{w}}=\frac{\rho_{w} \omega^{2}}{4 \pi}\left[\frac{1}{r} e^{-k_{w} r}-\frac{1}{r_{1}} e^{-k_{w} r_{1}}\right] \\
& \hat{q}_{n}^{G_{w}}=\frac{1}{4 \pi} \frac{\partial}{\partial \mathbf{n}(\xi)}\left[\frac{1}{r} e^{-k_{w} r}-\frac{1}{r_{1}} e^{-k_{w} r_{1}}\right]
\end{aligned}
$$

where

$$
\begin{aligned}
& r=\sqrt{\left(\xi_{j}-x_{j}\right)\left(\xi_{j}-x_{j}\right)}, \\
& r_{1}=\sqrt{\left(\xi_{1}-x\right)^{2}+\left(\xi_{2}-y\right)^{2}+\left(\xi_{3}+z\right)^{2}} .
\end{aligned}
$$

Note that the Green's function $\hat{p}_{w}^{G_{w}}$ fulfills the free surface condition automatically. Thus, the discretization along the surface of the seawater is avoided.

The boundary integral equation (54) also contains the Cauchy principal value integral. To eliminate the Cauchytype singularity in the boundary integral equation (54), we introduce the following boundary integral equation 


$$
\begin{aligned}
c^{(a)} \hat{p}_{w}^{(a)}(\mathbf{x}) & =\int_{\Gamma}\left[\hat{p}_{w}^{G_{a}}(\mathbf{x}, \boldsymbol{\xi}) \hat{q}_{n}^{(a)}(\boldsymbol{\xi})\right. \\
& \left.-\hat{q}_{n}^{G_{a}}(\mathbf{x}, \boldsymbol{\xi}) \hat{p}_{w}^{(a)}(\boldsymbol{\xi})\right] d \Gamma(\boldsymbol{\xi})
\end{aligned}
$$

with the Green's function given by

$$
\hat{p}_{w}^{G_{a}}=\frac{\rho_{w} \omega^{2}}{4 \pi}\left[\frac{1}{r}-\frac{1}{r_{1}}\right], \hat{q}_{n}^{G_{a}}=\frac{1}{4 \pi} \frac{\partial}{\partial \mathbf{n}(\xi)}\left[\frac{1}{r}-\frac{1}{r_{1}}\right]
$$

It is noticed that the singularity of the Green's function list in (58) is the same as that in (55)-(56) and also, the interior domain of the boundary integral equation (57) is opposite to that of the boundary integral equation (54). Thus, the outward normal vector for the boundary of (57) is opposite to that of (54). If solution $\hat{p}_{w}(\mathbf{x})$ at a point $\mathbf{X}$ of (54) is specified as a constant solution for (57), then, the following equation holds

$$
c^{(a)} \hat{p}_{w}(\mathbf{x})=\int_{\Gamma} \hat{q}_{n}^{G_{a}}(\mathbf{x}, \boldsymbol{\xi}) \hat{p}_{w}(\mathbf{x}) d \Gamma(\boldsymbol{\xi})
$$

Note that the sign of $\hat{q}_{n}^{G_{a}}(\mathbf{x}, \boldsymbol{\xi})$ in (59) is reversed from (57) to keep consistent with the sign convention of (54). Combination of equations (54) and (59) and consideration of the identity $c+c^{(a)}=1$ yield the following boundary integral equation

$$
\begin{aligned}
\hat{p}_{w}(\mathbf{x})= & \int_{\Gamma}\left\{\hat{p}_{w}^{G_{w}}(\mathbf{x}, \boldsymbol{\xi}) \hat{q}_{n}(\boldsymbol{\xi})-\left[\hat{q}_{n}^{G_{w}}(\mathbf{x}, \boldsymbol{\xi}) \hat{p}_{w}(\boldsymbol{\xi})\right.\right. \\
& \left.\left.-\hat{q}_{n}^{G_{a}}(\mathbf{x}, \boldsymbol{\xi}) \hat{p}_{w}(\mathbf{x})\right]\right\} d \Gamma(\boldsymbol{\xi})
\end{aligned}
$$

As the singularity of the Green's function $\hat{q}^{G_{w}}$ is the same as that of $\hat{q}^{G_{a}}$ when $\boldsymbol{\xi} \rightarrow \mathbf{x}$, the Cauchy-type singularity has been eliminated from equation (60). Therefore, after discretization of the boundary integral equation, the numerical integration over each boundary element can be performed directly by conventional quadrature formula.

\section{BEM FORMULATIONS FOR THE PILE, THE SEA- BED AND THE SEAWATER}

In this section, the boundary element method for the poro-elastic medium will be used to obtain the boundary element formulation for the pile and the seabed. The boundary element formulation for the seawater is obtained via the boundary element method for the acoustic medium.

To make our derivation more readable, the following notation for the boundaries of the system is introduced. As shown in Fig. 1, the interfaces between the pile, the seabed and the seawater are represented by $\Gamma_{1}, \Gamma_{2}, \Gamma_{4}$ and the interface between the pile and the air is denoted by $\Gamma_{3}$. Boundaries of the pile in contact with interfaces $\Gamma_{1}, \Gamma_{2}$ are denoted by $P_{1}, P_{2}$ and the boundary of the pile coincides with $\Gamma_{3}$ is represented by $P_{3}$. For the seabed, boundaries of the seabed in contact with interfaces $\Gamma_{1}, \Gamma_{4}$ are denoted by $S_{1}, S_{4}$, respectively, while for the seawater, boundaries along the interfaces $\Gamma_{2}, \Gamma_{4}$ are represented by $W_{2}, W_{4}$, respectively.

\section{BEM Formulations For The Pile}

As the pile is treated as a poro-elastic medium, thus, discretization of the boundary of the pile by certain number of boundary elements and application of the boundary integral equation (51) on the pile boundary yield the following equation

$$
\mathbf{G}^{(P)} \cdot \hat{\mathbf{t}}_{(P)}=\mathbf{H}^{(P)} \cdot \hat{\mathbf{u}}_{(P)}
$$

where the superscript $\mathrm{P}$ denotes the pile; $\mathbf{G}^{(P)}$ and $\mathbf{H}^{(P)}$ in (61) are the coefficient matrix obtained by integrating shape function kernel products over all the boundary elements of the pile; $\hat{\mathbf{u}}_{(P)}$ and $\hat{\mathbf{t}}_{(P)}$ are the generalized displacement and traction vector of the pile for all the nodes of the pile's boundary elements. Multiplying both side of equation (61) by $\mathbf{G}^{(P)^{-1}}$ and letting $\mathbf{E}^{(P)}=\mathbf{G}^{(P)^{-1}} \mathbf{H}^{(P)}$, one has

$$
\hat{\mathbf{t}}^{(P)}=\mathbf{E}^{(P)} \cdot \hat{\mathbf{u}}^{(P)}
$$

Dividing $\hat{\mathbf{u}}_{(P)}$ and $\hat{\mathbf{t}}_{(P)}$ into three parts corresponding to $P_{1}, P_{2}$ and $P_{3}$ [Fig. (1)], respectively, and rearranging the coefficient matrix $\mathbf{E}^{(P)}$ in (62), one has the following partitioned matrix equation

$$
\left\{\begin{array}{l}
\hat{\mathbf{t}}_{\left(P_{1}\right)} \\
\hat{\mathbf{t}}_{\left(P_{2}\right)} \\
\hat{\mathbf{t}}_{\left(P_{3}\right)}
\end{array}\right\}=\left[\begin{array}{lll}
\mathbf{E}_{11}^{(P)} & \mathbf{E}_{12}^{(P)} & \mathbf{E}_{13}^{(P)} \\
\mathbf{E}_{21}^{(P)} & \mathbf{E}_{22}^{(P)} & \mathbf{E}_{23}^{(P)} \\
\mathbf{E}_{31}^{(P)} & \mathbf{E}_{32}^{(P)} & \mathbf{E}_{33}^{(P)}
\end{array}\right] \times\left\{\begin{array}{c}
\hat{\mathbf{u}}_{\left(P_{1}\right)} \\
\hat{\mathbf{u}}_{\left(P_{2}\right)} \\
\hat{\mathbf{u}}_{\left(P_{3}\right)}
\end{array}\right\}
$$

where the subscripts $P_{1}, P_{2}$ and $P_{3}$ represent the parts of the pile boundary (Fig. 1); $\hat{\mathbf{u}}_{\left(P_{i}\right)}, \hat{\mathbf{t}}_{\left(P_{i}\right)}, \quad i=1,2,3$ denote the generalized displacement and traction vectors for the boundary $P_{1}, P_{2}$ and $P_{3}$ of the pile, respectively and have the following expressions

$$
\begin{gathered}
\hat{\mathbf{u}}_{\left(P_{i}\right)}=\left[\hat{\mathbf{u}}_{x\left(P_{i}\right)}, \hat{\mathbf{u}}_{y\left(P_{i}\right)}, \hat{\mathbf{u}}_{z\left(P_{i}\right)}, \hat{\mathbf{p}}_{\left(P_{i}\right)}\right]^{T} \\
=\left[\hat{u}_{x\left(P_{i}\right)}(1), \hat{u}_{x\left(P_{i}\right)}(2), \ldots, \hat{u}_{x\left(P_{i}\right)}\left(N_{P_{i}}\right) ;\right. \\
\hat{u}_{y\left(P_{i}\right)}(1), \ldots, \hat{u}_{y\left(P_{i}\right)}\left(N_{P_{i}}\right) ; \\
\hat{u}_{z\left(P_{i}\right)}(1), \ldots, \hat{u}_{z\left(P_{i}\right)}\left(N_{P_{i}}\right) ; \\
\left.\hat{p}_{\left(P_{i}\right)}(1), \ldots, \hat{p}_{\left(P_{i}\right)}\left(N_{P_{i}}\right)\right]^{T} \\
i=1,2,3 \\
\hat{\mathbf{t}}_{\left(P_{i}\right)}=\left[\hat{\mathbf{t}}_{x\left(P_{i}\right)}, \hat{\mathbf{t}}_{y\left(P_{i}\right)}, \hat{\mathbf{t}}_{z\left(P_{i}\right)}, \hat{\mathbf{w}}_{n\left(P_{i}\right)}\right] \\
=\left[\hat{t}_{x\left(P_{i}\right)}(1), \hat{t}_{x\left(P_{i}\right)}(2), \ldots, \hat{t}_{x\left(P_{i}\right)}\left(N_{P_{i}}\right) ;\right. \\
\hat{t}_{y\left(P_{i}\right)}(1), \ldots, \hat{t}_{y\left(P_{i}\right)}\left(N_{P_{i}}\right) ; \\
\hat{t}_{z\left(P_{i}\right)}(1), \ldots, \hat{t}_{z\left(P_{i}\right)}\left(N_{P_{i}}\right) ; \\
\left.\hat{w}_{n\left(P_{i}\right)}(1), \ldots, \hat{w}_{n\left(P_{i}\right)}\left(N_{P_{i}}\right)\right]^{T} \\
i=1,2,3
\end{gathered}
$$

where $N_{P i}$ is the total node number for the boundary $P_{i}$. 


\section{BEM Formulations For The Seabed}

Similarly, using the boundary integral equation (51) along the boundary of the seabed and following the similar procedure, a partitioned matrix equation can also be established for the seabed

$$
\left\{\begin{array}{l}
\hat{\mathbf{t}}_{\left(S_{1}\right)} \\
\hat{\mathbf{t}}_{\left(S_{4}\right)}
\end{array}\right\}=\left[\begin{array}{ll}
\mathbf{E}_{11}^{(S)} & \mathbf{E}_{14}^{(S)} \\
\mathbf{E}_{41}^{(S)} & \mathbf{E}_{44}^{(S)}
\end{array}\right] \cdot\left\{\begin{array}{l}
\hat{\mathbf{u}}_{\left(S_{1}\right)} \\
\hat{\mathbf{u}}_{\left(S_{4}\right)}
\end{array}\right\}
$$

where $\hat{\mathbf{u}}_{\left(S_{i}\right)}, \hat{\mathbf{t}}_{\left(S_{i}\right)}, i=1,4$ denote the generalized displacement and traction vectors of the seabed boundaries $S_{1}$ and $S_{4}$ (Fig. 1), which have similar elements arrangement as in (64)-(65).

\section{BEM Formulations For The Seawater}

Application of the boundary integral equation (60) and implementation the discretization scheme corresponding to the pile and the seabed, the following partitioned matrix equation is obtained for the seawater

$$
\left\{\begin{array}{l}
\hat{\mathbf{q}}_{n\left(W_{2}\right)} \\
\hat{\mathbf{q}}_{n\left(W_{4}\right)}
\end{array}\right\}=\left[\begin{array}{ll}
\mathbf{E}_{22}^{(W)} & \mathbf{E}_{24}^{(W)} \\
\mathbf{E}_{42}^{(W)} & \mathbf{E}_{44}^{(W)}
\end{array}\right] \times\left\{\begin{array}{l}
\hat{\mathbf{p}}_{w\left(W_{2}\right)} \\
\hat{\mathbf{p}}_{w\left(W_{4}\right)}
\end{array}\right\}
$$

where $\hat{\mathbf{q}}_{n\left(W_{i}\right)}, \hat{\mathbf{p}}_{w\left(W_{i}\right)}, \quad i=2,4$ denote the normal displacement and the pressure of the seawater along the boundary $W_{2}$ and $W_{4}$ and have the following form

$$
\begin{aligned}
& \hat{\mathbf{q}}_{n\left(W_{i}\right)}=\left[\hat{q}_{n\left(W_{i}\right)}(1), \hat{q}_{n\left(W_{i}\right)}(2), \ldots, \hat{q}_{n\left(W_{i}\right)}\left(N_{W_{i}}\right)\right]^{T}, \\
& \hat{\mathbf{p}}_{w\left(W_{i}\right)}=\left[\hat{p}_{w\left(W_{i}\right)}(1), \hat{p}_{w\left(W_{i}\right)}(2), \ldots, \hat{p}_{w\left(W_{i}\right)}\left(N_{W_{i}}\right)\right]^{T}, \quad i=2,4
\end{aligned}
$$

where $\hat{q}_{n\left(W_{i}\right)}(j), \hat{p}_{w\left(W_{i}\right)}(j)$ denote the normal displacement and the pressure at the $\mathrm{j}$-th node of the boudnary $W_{i}$

\section{THE COUPLED BEM MODEL FOR THE PILE, THE SEABED AND THE SEAWATER}

In above section, three boundary element formulations are established for the pile, the seabed and the seawater. In this section, the three boundary element formulations and the continuity conditions along the interfaces between the pile, the seabed and the seawater are used to establish the coupled boundary element model for the system.

\section{The Continuity Conditions Between Three Domains}

The continuity conditions at the interface $\Gamma_{1}$ between the pile and the seabed (Fig. (1)) have the following form [25]

$$
\hat{\mathbf{u}}_{\left(P_{1}\right)}=\hat{\mathbf{u}}_{\left(S_{1}\right)}, \hat{\mathbf{t}}_{\left(P_{1}\right)}=-\hat{\mathbf{t}}_{\left(S_{1}\right)}
$$

The continuity conditions at the interface $\Gamma_{2}$ between the pile and the seawater have the following form [26]

$$
\begin{aligned}
& \hat{\mathbf{p}}_{\left(P_{2}\right)}=\hat{\mathbf{p}}_{\left(W_{2}\right)}, \\
& \hat{\mathbf{u}}_{n\left(P_{2}\right)}+\hat{\mathbf{w}}_{n\left(P_{2}\right)}=-\hat{\mathbf{q}}_{n\left(W_{2}\right)}, \\
& \hat{\mathbf{t}}_{x\left(P_{2}\right)}+\mathbf{n}_{x\left(P_{2}\right)} \cdot \hat{\mathbf{p}}_{\left(W_{2}\right)}=\hat{\mathbf{t}}_{x\left(P_{2}\right)}+\mathbf{n}_{x\left(P_{2}\right)} \cdot \hat{\mathbf{p}}_{\left(P_{2}\right)}=\overline{\mathbf{t}}_{x\left(P_{2}\right)}, \\
& \hat{\mathbf{t}}_{y\left(P_{2}\right)}+\mathbf{n}_{y\left(P_{2}\right)} \cdot \hat{\mathbf{p}}_{\left(W_{2}\right)}=\hat{\mathbf{t}}_{y\left(P_{2}\right)}+\mathbf{n}_{y\left(P_{2}\right)} \cdot \hat{\mathbf{p}}_{\left(P_{2}\right)}=\overline{\hat{\mathbf{t}}}_{y\left(P_{2}\right)},
\end{aligned}
$$

$$
\hat{\mathbf{t}}_{z\left(P_{2}\right)}=\mathbf{0}
$$

where $\hat{\mathbf{p}}_{\left(P_{2}\right)}, \hat{\mathbf{u}}_{n\left(P_{2}\right)}$ and $\hat{\mathbf{w}}_{n\left(P_{2}\right)}$ are the pore pressure, the normal displacement of the solid frame and the normal infiltration displacement of the pore fluid along the boundary $P_{2}$ of the pile; $\hat{\mathbf{t}}_{x\left(P_{2}\right)}, \hat{\mathbf{t}}_{y\left(P_{2}\right)}, \hat{\mathbf{t}}_{z\left(P_{2}\right)}$ are the traction applied to the pile boundary $P_{2}$ in the $\mathrm{x}, \mathrm{y}$ and $\mathrm{z}$ direction, respectively and $\overline{\hat{\mathbf{t}}}_{x\left(P_{2}\right)}, \overline{\hat{\mathbf{t}}}_{y\left(P_{2}\right)}$ are the external tractions exerted on the boundary $P_{2}$ in the $\mathrm{x}, \mathrm{y}$ direction due to the water wave; $\mathbf{n}_{x\left(P_{2}\right)}$, $\mathbf{n}_{y\left(P_{2}\right)}$ are the direction cosine matrix in the $\mathrm{x}$ and $\mathrm{y}$ direction along the pile boundary $P_{2}$. The direction cosine matrix $\mathbf{n}_{x\left(P_{i}\right)}, \mathbf{n}_{y\left(P_{2}\right)}$ for the $P_{i}$ boundary of the pile are arranged in the following matrix form

$$
\mathbf{n}_{j\left(P_{i}\right)}=\left[\begin{array}{lccc}
n_{j\left(P_{i}\right)}(1) & 0 & \ldots \ldots & 0 \\
0 & n_{j\left(P_{i}\right)}(2) & \ldots . . & 0 \\
\ldots . . & & & \\
0 & 0 \ldots \ldots . & & n_{j\left(P_{i}\right)}\left(N_{P_{i}}\right)
\end{array}\right]_{N_{P_{i}} \times N_{P_{i}}}
$$

The continuity conditions between the seabed and the seawater at the interface $\Gamma_{4}$ (Fig. 1) have the following form [26]

$$
\begin{aligned}
& \hat{\mathbf{p}}_{\left(S_{4}\right)}=\hat{\mathbf{p}}_{\left(W_{4}\right)}, \\
& -\hat{\mathbf{u}}_{z\left(S_{4}\right)}+\hat{\mathbf{w}}_{n\left(S_{4}\right)}=-\hat{\mathbf{q}}_{n\left(W_{4}\right)}, \\
& \hat{\mathbf{t}}_{x\left(S_{4}\right)}=\mathbf{0}, \\
& \hat{\mathbf{t}}_{y\left(S_{4}\right)}=\mathbf{0}, \\
& \hat{\mathbf{t}}_{z\left(S_{4}\right)}-\hat{\mathbf{p}}_{\left(W_{4}\right)}=\hat{\mathbf{t}}_{z\left(S_{4}\right)}-\hat{\mathbf{p}}_{\left(S_{4}\right)}=\overline{\hat{\mathbf{t}}} z\left(S_{4}\right)
\end{aligned}
$$

where $\overline{\hat{\mathbf{t}}}_{z\left(S_{4}\right)}$ is the external traction applied to the boundary $S_{4}$ in the z direction due to the water wave.

\section{The Coupled BEM Model For The System}

Using the continuity condition (69) and equations (63) and(66), the following equation along the interface $\Gamma_{1}$ is obtained

$$
\begin{aligned}
\left(\mathbf{E}_{11}^{(P)}+\mathbf{E}_{11}^{(S)}\right) \cdot \hat{\mathbf{u}}_{\left(S_{1}\right)} & +\mathbf{E}_{12}^{(P)} \cdot \hat{\mathbf{u}}_{\left(P_{2}\right)}+\mathbf{E}_{13}^{(P)} \cdot \hat{\mathbf{u}}_{\left(P_{3}\right)} \\
& +\mathbf{E}_{14}^{(S)} \cdot \hat{\mathbf{u}}_{\left(S_{4}\right)}=\mathbf{0}
\end{aligned}
$$

The above equation can be rewritten in the following matrix form

$$
\left[\left(\mathbf{E}_{11}^{(P)}+\mathbf{E}_{11}^{(S)}\right), \mathbf{E}_{12}^{(P)}, \mathbf{E}_{13}^{(P)}, \mathbf{E}_{14}^{(S)}\right] \times\left\{\begin{array}{c}
\hat{\mathbf{u}}_{\left(S_{1}\right)} \\
\hat{\mathbf{u}}_{\left(P_{2}\right)} \\
\hat{\mathbf{u}}_{\left(P_{3}\right)} \\
\hat{\mathbf{u}}_{\left(S_{4}\right)}
\end{array}\right\}=\mathbf{0}
$$


Using the BEM formulation for the pile, the traction $\hat{\mathbf{t}}_{(P,)}$ along the boundary $P_{2}$ of the pile is expressed in the following form

$$
\begin{array}{r}
\hat{\mathbf{t}}_{\left(P_{2}\right)}=\left[\mathbf{E}_{21}^{(P)}, \mathbf{E}_{22}^{(P)}, \mathbf{E}_{23}^{(P)}\right] \times\left[\hat{\mathbf{u}}_{\left(P_{1}\right)}, \hat{\mathbf{u}}_{\left(P_{2}\right)}, \hat{\mathbf{u}}_{\left(P_{3}\right)}\right]^{T} \\
=\left[\mathbf{E}_{21}^{(P)}, \mathbf{E}_{22}^{(P)}, \mathbf{E}_{23}^{(P)}, \mathbf{0}\right] \times\left\{\begin{array}{c}
\hat{\mathbf{u}}_{\left(S_{1}\right)} \\
\hat{\mathbf{u}}_{\left(P_{2}\right)} \\
\hat{\mathbf{u}}_{\left(P_{3}\right)} \\
\hat{\mathbf{u}}_{\left(S_{4}\right)}
\end{array}\right\}
\end{array}
$$

On the other hand, the traction along the boundary $P_{2}$ of the pile can have the following expression if the continuity condition (70)-(74) is taken into account

$$
\begin{aligned}
& \hat{\mathbf{t}}_{\left(P_{2}\right)}=\left\{\begin{array}{c}
-\mathbf{n}_{x\left(P_{2}\right)} \cdot \hat{\mathbf{p}}_{w\left(W_{2}\right)}+\overline{\hat{\mathbf{t}}}_{x\left(P_{2}\right)} \\
-\mathbf{n}_{y\left(P_{2}\right)} \cdot \hat{\mathbf{p}}_{w\left(W_{2}\right)}+\overline{\hat{\mathbf{t}}}_{y\left(P_{2}\right)} \\
\mathbf{0} \\
\hat{\mathbf{w}}_{n\left(P_{2}\right)}
\end{array}\right\} \\
& =\left\{\begin{array}{c}
-\mathbf{n}_{x\left(P_{2}\right)} \cdot \hat{\mathbf{p}}_{\left(P_{2}\right)}+\overline{\hat{\mathbf{t}}}_{x\left(P_{2}\right)} \\
-\mathbf{n}_{y\left(P_{2}\right)} \cdot \hat{\mathbf{p}}_{\left(P_{2}\right)}+\overline{\hat{\mathbf{t}}}_{y\left(P_{2}\right)} \\
\mathbf{0} \\
-\left[\mathbf{n}_{x\left(P_{2}\right)} \cdot \hat{\mathbf{u}}_{x\left(P_{2}\right)}+\mathbf{n}_{y\left(P_{2}\right)} \cdot \hat{\mathbf{u}}_{y\left(P_{2}\right)}\right]-\hat{\mathbf{q}}_{n\left(W_{2}\right)}
\end{array}\right\}
\end{aligned}
$$

Using the BEM formulation for the seawater, the normal displacement of the seawater along the boundary $W_{2}$ has the following form

$$
\begin{array}{r}
\hat{\mathbf{q}}_{n\left(W_{2}\right)}=\mathbf{E}_{22}^{(W)} \cdot \hat{\mathbf{p}}_{w\left(W_{2}\right)}+\mathbf{E}_{24}^{(W)} \cdot \hat{\mathbf{p}}_{w\left(W_{4}\right)} \\
=\mathbf{E}_{22}^{(W)} \cdot \hat{\mathbf{p}}_{\left(P_{2}\right)}+\mathbf{E}_{24}^{(W)} \cdot \hat{\mathbf{p}}_{\left(S_{4}\right)}
\end{array}
$$

Using equations (84) and (85), one has the following equation for $\mathbf{t}_{\left(P_{2}\right)}$

$$
\begin{aligned}
& \hat{\mathbf{t}}_{\left(P_{2}\right)}= \\
& \left\{\begin{array}{c}
-\mathbf{n}_{x\left(P_{2}\right)} \cdot \hat{\mathbf{p}}_{\left(P_{2}\right)} \\
-\mathbf{n}_{y\left(P_{2}\right)} \cdot \hat{\mathbf{p}}_{\left(P_{2}\right)} \\
\mathbf{0} \\
-\left[\mathbf{n}_{x\left(P_{2}\right)} \cdot \hat{\mathbf{u}}_{x\left(P_{2}\right)}+\mathbf{n}_{y\left(P_{2}\right)} \cdot \hat{\mathbf{u}}_{y\left(P_{2}\right)}\right]-\left[\mathbf{E}_{22}^{(W)} \cdot \hat{\mathbf{p}}_{\left(P_{2}\right)}+\mathbf{E}_{24}^{(W)} \cdot \hat{\mathbf{p}}_{\left(S_{4}\right)}\right]
\end{array}\right\} \\
& +\left\{\begin{array}{c}
\overline{\hat{\mathbf{t}}}_{x\left(P_{2}\right)} \\
\overline{\hat{\mathbf{t}}}_{y\left(P_{2}\right)} \\
\mathbf{0} \\
\mathbf{0}
\end{array}\right\}=\left[\begin{array}{llll}
\mathbf{0} & \mathbf{A}_{(P W)} & \mathbf{0} & \mathbf{B}_{(P W)}
\end{array}\right] \times\left\{\begin{array}{c}
\hat{\mathbf{u}}_{\left(S_{1}\right)} \\
\hat{\mathbf{u}}_{\left(P_{2}\right)} \\
\hat{\mathbf{u}}_{\left(P_{3}\right)} \\
\hat{\mathbf{u}}_{\left(S_{4}\right)}
\end{array}\right]+\left\{\begin{array}{c}
\overline{\hat{\mathbf{t}}}_{x\left(P_{2}\right)} \\
\overline{\mathbf{t}}_{y\left(P_{2}\right)} \\
\mathbf{0} \\
\mathbf{0}
\end{array}\right\}
\end{aligned}
$$

where

$$
\mathbf{A}_{(P W)}=\left[\begin{array}{cccc}
\mathbf{0} & \mathbf{0} & \mathbf{0} & -\mathbf{n}_{x\left(P_{2}\right)} \\
\mathbf{0} & \mathbf{0} & \mathbf{0} & -\mathbf{n}_{y\left(P_{2}\right)} \\
\mathbf{0} & \mathbf{0} & \mathbf{0} & \mathbf{0} \\
-\mathbf{n}_{x\left(P_{2}\right)} & -\mathbf{n}_{y\left(P_{2}\right)} & \mathbf{0} & -\mathbf{E}_{22}^{(W)}
\end{array}\right]
$$

$$
\mathbf{B}_{(P W)}=\left[\begin{array}{cccc}
\mathbf{0} & \mathbf{0} & \mathbf{0} \\
\mathbf{0} & 0 & \mathbf{0} & \mathbf{0} \\
\mathbf{0} & 0 & 0 & \mathbf{0} \\
\mathbf{0} & 0 & \mathbf{0} & -\mathbf{E}_{24}^{(W)}
\end{array}\right]
$$

Combination of equations (83) and (86) gives the following coupling equation for the seawater and the pile along the interface $\Gamma_{2}$

$$
\begin{aligned}
& {\left[\mathbf{E}_{21}^{(P)}, \mathbf{E}_{22}^{(P)}-\mathbf{A}_{(P W)}, \mathbf{E}_{23}^{(P)},-\mathbf{B}_{(P W)}\right] \times\left\{\begin{array}{c}
\hat{\mathbf{u}}_{\left(S_{1}\right)} \\
\hat{\mathbf{u}}_{\left(P_{2}\right)} \\
\hat{\mathbf{u}}_{\left(P_{3}\right)} \\
\hat{\mathbf{u}}_{\left(S_{4}\right)}
\end{array}\right\} } \\
&=\left\{\begin{array}{c}
\overline{\hat{\mathbf{t}}}_{x\left(P_{2}\right)} \\
\hat{\mathbf{t}}_{y\left(P_{2}\right)} \\
\mathbf{0} \\
\mathbf{0}
\end{array}\right\}
\end{aligned}
$$

For the boundary $\Gamma_{3}$ of the pile, it is assumed that $\hat{\mathbf{t}}_{\left(P_{3}\right)}$ is known a prior and thus using (63), one has the following equation for the interface $\Gamma_{3}$

$$
\left[\mathbf{E}_{31}^{(P)}, \mathbf{E}_{32}^{(P)}, \mathbf{E}_{33}^{(P)}\right] \cdot\left[\hat{\mathbf{u}}_{\left(P_{1}\right)}, \hat{\mathbf{u}}_{\left(P_{2}\right)}, \hat{\mathbf{u}}_{\left(P_{3}\right)}\right]^{T}=\overline{\hat{\mathbf{t}}}_{\left(P_{3}\right)}
$$

where $\overline{\hat{\mathbf{t}}}_{\left(P_{3}\right)}$ is the given traction along the boundary $P_{3}$ of the pile. The above equation can be rearranged into the following matrix form

$$
\left[\mathbf{E}_{31}^{(P)}, \mathbf{E}_{32}^{(P)}, \mathbf{E}_{33}^{(P)}, \mathbf{0}\right] \times\left\{\begin{array}{c}
\hat{\mathbf{u}}_{\left(S_{1}\right)} \\
\hat{\mathbf{u}}_{\left(P_{2}\right)} \\
\hat{\mathbf{u}}_{\left(P_{3}\right)} \\
\hat{\mathbf{u}}_{\left(S_{4}\right)}
\end{array}\right\}=\overline{\hat{\mathbf{t}}}_{\left(P_{3}\right)}
$$

According to the BEM formulation for the seabed (66), the traction along the boundary $S_{4}$ of the seabed has the form

$$
\hat{\mathbf{t}}_{\left(S_{4}\right)}=\mathbf{E}_{41}^{(S)} \cdot \hat{\mathbf{u}}_{\left(S_{1}\right)}+\mathbf{E}_{44}^{(S)} \cdot \hat{\mathbf{u}}_{\left(S_{4}\right)}
$$

On the other hand, in terms of the continuity condition (76)-(80) along $\Gamma_{4}$, the traction along the boundary $S_{4}$ of the seabed may be expressed as

$$
\begin{aligned}
\hat{\mathbf{t}}_{\left(S_{4}\right)}=\left\{\begin{array}{c}
\hat{\mathbf{t}}_{x\left(S_{4}\right)} \\
\hat{\mathbf{t}}_{y\left(S_{4}\right)} \\
\hat{\mathbf{t}}_{z\left(S_{4}\right)} \\
\hat{\mathbf{w}}_{n\left(S_{4}\right)}
\end{array}\right\}= \\
=\left\{\begin{array}{c}
\mathbf{0} \\
\mathbf{0} \\
\hat{\mathbf{p}}_{w\left(\left(_{4}\right)\right.}+\overline{\hat{\mathbf{t}}}_{z\left(S_{4}\right)} \\
\hat{\mathbf{w}}_{n\left(S_{4}\right)}
\end{array}\right\} \\
=\left\{\begin{array}{c}
\mathbf{0} \\
\mathbf{0} \\
\hat{\mathbf{p}}_{\left(S_{4}\right)}+\overline{\hat{\mathbf{t}}}_{z\left(S_{4}\right)} \\
\hat{\mathbf{u}}_{z\left(S_{4}\right)}-\hat{\mathbf{q}}_{n\left(W_{4}\right)}
\end{array}\right\}
\end{aligned}
$$


The normal displacement of the seawater $\hat{\mathbf{q}}_{n\left(W_{4}\right)}$ along the boundary $S_{4}$ of the seabed can be obtained using the BEM formulation (67) for the seawater, which gives

$$
\begin{aligned}
\hat{\mathbf{q}}_{n\left(W_{4}\right)}= & \mathbf{E}_{42}^{(W)} \cdot \hat{\mathbf{p}}_{w\left(W_{2}\right)}+\mathbf{E}_{44}^{(W)} \cdot \hat{\mathbf{p}}_{w\left(W_{4}\right)} \\
= & \mathbf{E}_{42}^{(W)} \cdot \hat{\mathbf{p}}_{\left(P_{2}\right)}+\mathbf{E}_{44}^{(W)} \cdot \hat{\mathbf{p}}_{\left(S_{4}\right)}
\end{aligned}
$$

Substitution of the above equation into (92) yields the following equation

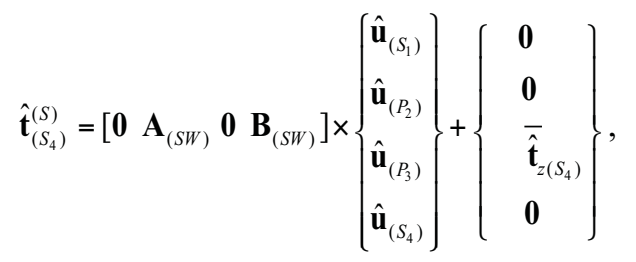

in which

$$
\begin{aligned}
\mathbf{A}_{(S W)} & =\left[\begin{array}{ccc}
\mathbf{0 0} & 0 & \mathbf{0} \\
\mathbf{0 0} & 0 & \mathbf{0} \\
\mathbf{0 0} & 0 & \mathbf{0} \\
\mathbf{0 0} & \mathbf{0} & -\mathbf{E}_{42}^{(W)}
\end{array}\right] \\
\mathbf{B}_{(S W)} & =\left[\begin{array}{lll}
\mathbf{0 0} & \mathbf{0} & \mathbf{0} \\
\mathbf{0 0} & \mathbf{0} & \mathbf{0} \\
\mathbf{0 0} & \mathbf{0} & \mathbf{I} \\
\mathbf{0 0} & \mathbf{I} & -\mathbf{E}_{44}^{(W)}
\end{array}\right],
\end{aligned}
$$

Combination of equation (94) and equation (91) gives the coupling equation along the interface $\Gamma_{4}$

$$
\left[\mathbf{E}_{41}^{(S)},-\mathbf{A}_{(S W)}, \mathbf{0}, \mathbf{E}_{44}^{(S)}-\mathbf{B}_{(S W)}\right] \times\left\{\begin{array}{c}
\hat{\mathbf{u}}_{\left(S_{1}\right)} \\
\hat{\mathbf{u}}_{\left(P_{2}\right)} \\
\hat{\mathbf{u}}_{\left(P_{3}\right)} \\
\hat{\mathbf{u}}_{\left(S_{4}\right)}
\end{array}\right\}=\left\{\begin{array}{c}
\mathbf{0} \\
\mathbf{0} \\
\overline{\hat{\mathbf{t}}}_{z\left(S_{4}\right)} \\
\mathbf{0}
\end{array}\right\}
$$

Finally, combination of equations (82), (88), (90) and (97) yields the following equations for the unknowns $\hat{\mathbf{u}}_{\left(S_{1}\right)}$,

$$
\hat{\mathbf{u}}_{\left(P_{2}\right)}, \hat{\mathbf{u}}_{\left(P_{3}\right)} \text { and } \hat{\mathbf{u}}_{\left(S_{4}\right)}
$$

$$
\begin{aligned}
& {\left[\begin{array}{lccc}
\mathbf{E}_{11}^{(P)}+\mathbf{E}_{11}^{(S)}, & \mathbf{E}_{12}^{(P)}, & \mathbf{E}_{13}^{(P)}, & \mathbf{E}_{14}^{(S)} \\
\mathbf{E}_{21}^{(P)}, & \mathbf{E}_{22}^{(P)}-\mathbf{A}_{(P W)}, & \mathbf{E}_{23}^{(P)}, & -\mathbf{B}_{(P W)} \\
\mathbf{E}_{31}^{(P)}, & \mathbf{E}_{32}^{(P)}, & \mathbf{E}_{33}^{(P)}, & \mathbf{0} \\
\mathbf{E}_{41}^{(S)}, & -\mathbf{A}_{(S W)}, & \mathbf{0}, & \mathbf{E}_{44}^{(S)}-\mathbf{B}_{(S W)}
\end{array}\right]} \\
& \times\left\{\begin{array}{l}
\hat{\mathbf{u}}_{\left(S_{1}\right)} \\
\hat{\mathbf{u}}_{\left(P_{2}\right)} \\
\hat{\mathbf{u}}_{\left(P_{3}\right)} \\
\hat{\mathbf{u}}_{\left(S_{4}\right)}
\end{array}\right\}=\left\{\begin{array}{l}
\mathbf{0} \\
\hat{\mathbf{R}}_{(P W)} \\
\overline{\mathbf{t}}_{\left(P_{3}\right)} \\
\hat{\mathbf{R}}_{(S W)}
\end{array}\right\}
\end{aligned}
$$

Where

$$
\hat{\mathbf{R}}_{(P W)}=\left\{\begin{array}{c}
\overline{\hat{\mathbf{t}}}_{x\left(P_{2}\right)} \\
\overline{\hat{\mathbf{t}}}_{y\left(P_{2}\right)} \\
\mathbf{0} \\
\mathbf{0}
\end{array}\right\}, \hat{\mathbf{R}}_{(S W)}=\left\{\begin{array}{l}
\mathbf{0} \\
\mathbf{0} \\
\overline{\hat{\mathbf{t}}}_{z\left(S_{4}\right)} \\
\mathbf{0}
\end{array}\right\}
$$

It is worth noting once $\hat{\mathbf{u}}_{\left(S_{1}\right)}, \hat{\mathbf{u}}_{\left(P_{2}\right)}, \hat{\mathbf{u}}_{\left(P_{3}\right)}$ and $\hat{\mathbf{u}}_{\left(S_{4}\right)}$ are obtained by (98), unknown tractions along the interface $\Gamma_{1}$ can be evaluated by (63) or (66). Thus, all the unknown variables along the boundary of the system are determined. And variables within interior of the domains for the pile, the seabed and the seawater can be calculated using the corresponding Somigliana identities.

\section{NUMERICAL RESULTS}

In this section, numerical examples will be used to demonstrate the capacity of the proposed coupled model. In the examples, the influence of the modulus ratio between the pile and the seabed will be examined. The pile is assumed to have a circular cross section. The boundary of the pile, the seabed and the seawater is covered by 2-D eight-node isoparametric boundary elements. As the current pile-seabedseawater system is symmetrical with respect to yoz plane, only half of the boundary of the system needs to be discretized. The top and the bottom of the pile as well as the boundary of the seabed in contact with the bottom of the pile are discretized by the elements and the nodes as shown in Fig. (2). The side of the pile and the corresponding boundaries of the seabed and the seawater are divided into several segments and each segment is discretized by certain number of boundary elements which match the elements and the nodes at the bottom and the top of the pile. Note that to round off the corners of the seabed and the seawater, the boundary of the pile is divided into three parts and each pile boundary is separated by a very small separation at the interface of the seabed and the seawater as well as at the surface of the seawater. Moreover, the interface between the seabed and the seawater is discretized by a series of concentric annular rings, each of which is further discretized by the same number of boundary elements as each segment of the pile.

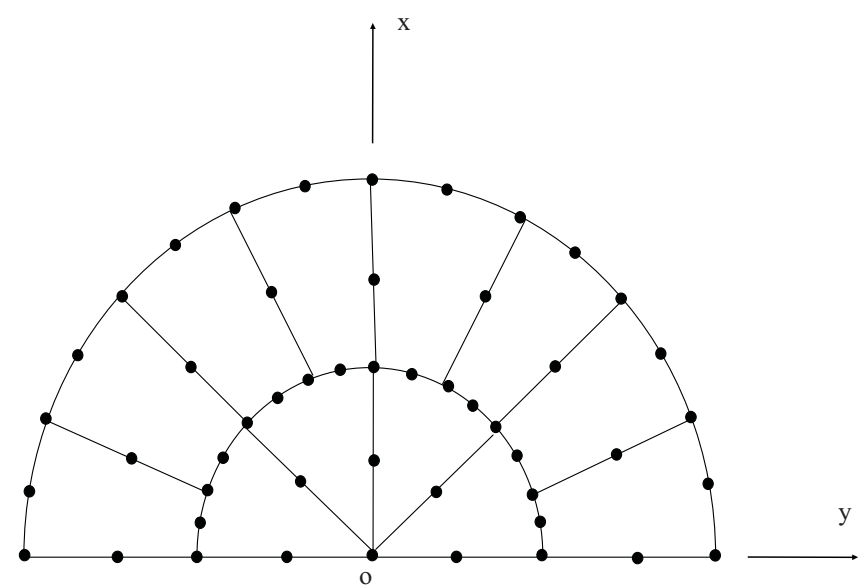

Fig. (2). Discretization scheme for the bottom, the top of the pile by twelve boundary elements and forty nine nodes. 
In this example, the embedded length of the pile in the seabed is $h_{1}=15 \mathrm{~m}$ and the depth of the overlying seawater is $h_{2}=15 \mathrm{~m}$. The length of the pile above the water surface is $h_{3}=1 \mathrm{~m}$ and the diameter of the pile is $d=0.6 \mathrm{~m}$ (Fig. (1)). As mentioned previously, the bottom and the top of the pile are discretized by twelve elements (Fig. (2)), respectively. The first part of the pile which is embedded in the seabed is divided into 15 segments uniformly and to match the discretization scheme of the bottom of the pile (Fig. (2)), each segment of the first part is discretized by eight elements. The second and the third part of the pile which are in contact with the seawater and above the seawater are divided into 15 segments and two segments uniformly and each segment of the second and the third part is also discretized by eight elements. Moreover, the infinite interface between the seabed and the seawater is simulated by twelve annular rings, and each ring is covered by eight boundary elements.

The traction $\overline{\hat{\mathbf{t}}}_{\left.P_{3}\right)}$ along the boundary $\Gamma_{3}$ of the pile is assumed to vanish. The angular frequencies of the incident water wave are $\omega=1,3,6$, respectively and the wave height of the incident water wave is assumed to be $3 \mathrm{~m}$. The parameters for the seabed and the seawater are given in Table (1). The Lame's constants $\mu_{p}$ and $\lambda_{p}$ of the pile take the vales: $\mu_{p} / \mu_{s}=\lambda_{p} / \lambda_{s}=100,300,1000$, respectively, where the subscripts $\mathrm{p}, \mathrm{s}$ denote the pile and the seabed, respectively. The other parameters of the pile assume the values as given in Table (1).

Table 1. The Parameters for the Seabed, the Pile and the Seawater in the Numerical Example

\begin{tabular}{|c|c|}
\hline Parameters & Values \\
\hline$\lambda(\mathrm{Pa})$ & $4.0 \times 10^{7} \quad$ (seabed) \\
\hline$\mu(\mathrm{Pa})$ & $2.0 \times 10^{7} \quad \mathrm{~Pa}$ (seabed) \\
\hline$\rho_{s}\left(\mathrm{~kg} / \mathrm{m}^{3}\right)$ & $\begin{array}{c}2.5 \times 10^{3} \mathrm{~kg} / \mathrm{m}^{3} \text { (seabed) } \\
\left.2.5 \times 10^{3} \mathrm{~kg} / \mathrm{m}^{3} \text { (pile }\right)\end{array}$ \\
\hline$\rho_{f}\left(\mathrm{~kg} / \mathrm{m}^{3}\right)$ & $\begin{array}{c}1.0 \times 10^{3} \mathrm{~kg} / \mathrm{m}^{3} \text { (seabed) }, \\
\left.\mathrm{kg} / \mathrm{m}^{3} \text { (pile }\right)\end{array}$ \\
\hline$\phi$ & 0.4 (seabed), 0.3 (pile) \\
\hline$a_{\infty}$ & 2 (seabed), 2 (pile) \\
\hline$\alpha$ & 0.8 (seabed), 0.8 (pile) \\
\hline$M(\mathrm{~Pa})$ & $\begin{array}{c}5.0 \times 10^{8} \text { ( seabed) } \\
2.0 \times 10^{10}(\text { pile })\end{array}$ \\
\hline$\eta$ (Pa.s) & $\begin{array}{c}1.0 \times 10^{-3} \text { ( seabed) } \\
1.0 \times 10^{-3}(\text { pile })\end{array}$ \\
\hline$k\left(\mathrm{~m}^{2}\right)$ & $\begin{array}{c}\left.1.0 \times 10^{-12} \text { ( seabed }\right) \\
1.0 \times 10^{-13} \text { ( pile) }\end{array}$ \\
\hline$\rho_{w}\left(\mathrm{~kg} / \mathrm{m}^{3}\right)$ & $1.0 \times 10^{3}$ \\
\hline$v_{w}(\mathrm{~m} / \mathrm{s})$ & 1414.0 \\
\hline
\end{tabular}

Figs. (3)-(5) plots the amplitudes for the horizontal displacement, the shear force of the pile and the pore pressure amplitudes at the nodes of the seabed in contact with the left and the right side of the pile for the cases of $\omega=1,3,6$, respectively. Note that the horizontal displacement of the pile at a given vertical coordinate is obtained by averaging the horizontal displacements of the elements' nodes at the same vertical coordinate, while the shear force of the pile is obtained by integrating the obtained boundary traction in $y$ direction along the boundary of the pile.

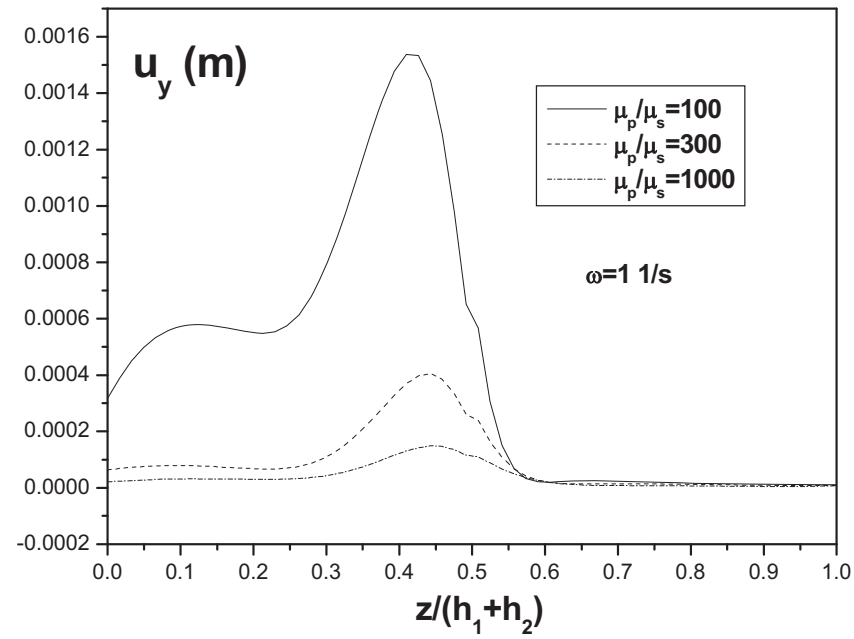

Fig. (3a). The influence of the modulus ratio between the pile and the seabed ( $\left.\mu_{p} / \mu_{s}, \lambda_{p} / \lambda_{s}\right)$ on the horizontal displacement $\left(u_{y}\right)$ for the case of $\omega=1[1 / \mathrm{s}]$.

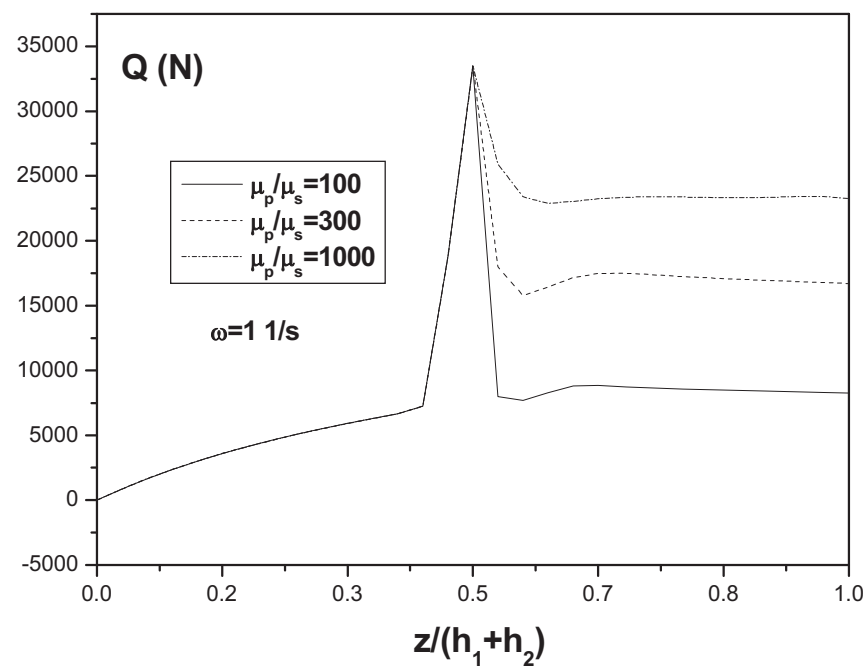

Fig. (3b). The influence of the modulus ratio between the pile and the seabed $\left(\mu_{p} / \mu_{s}, \lambda_{p} / \lambda_{s}\right)$ on the shear force of the pile (Q) for the case of $\omega=1[1 / \mathrm{s}]$.

Figs. 3 (a), 4 (a) and 5 (a) show that when the angular frequency of the incident water wave increases from $\omega=1$ to $\omega=3$, the horizontal displacement of the pile increase considerably. However, when the angular frequency varies from $\omega=3$ to $\omega=6$, the horizontal displacement of the pile decreases pronouncedly. Also, for all angular frequencies of the water wave, with increase modulus ratio, the horizontal displacement of the pile decreases significantly. Moreover, the horizontal displacement for the part of the pile embedded in the seabed, is much smaller that for the part immersed in the seawater. 


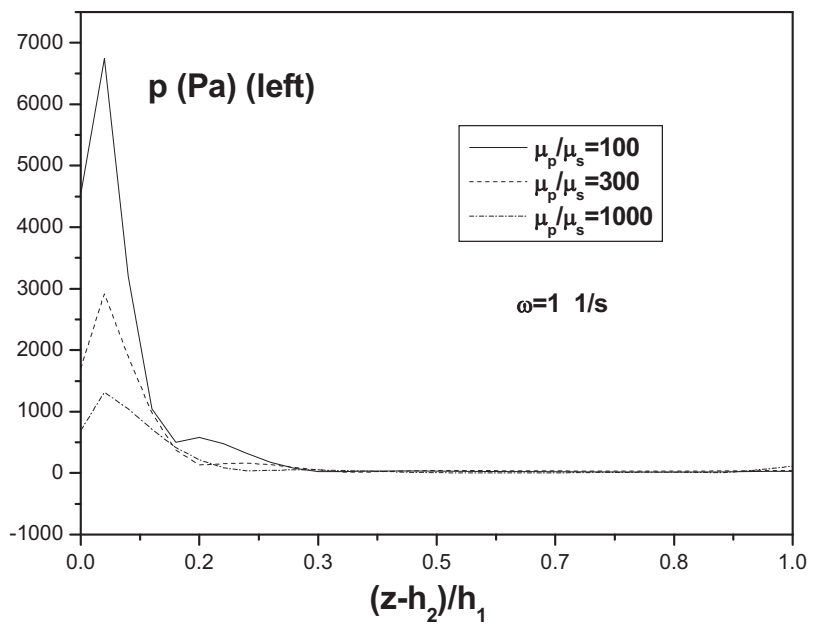

Fig. (3c). The influence of the modulus ratio between the pile and the seabed $\left(\mu_{p} / \mu_{s}, \lambda_{p} / \lambda_{s}\right)$ on the pore pressure of the seabed contacting the left side of the pile ( $p_{\text {left }}$ ) for the case of $\omega=1$ $[1 / \mathrm{s}]$.

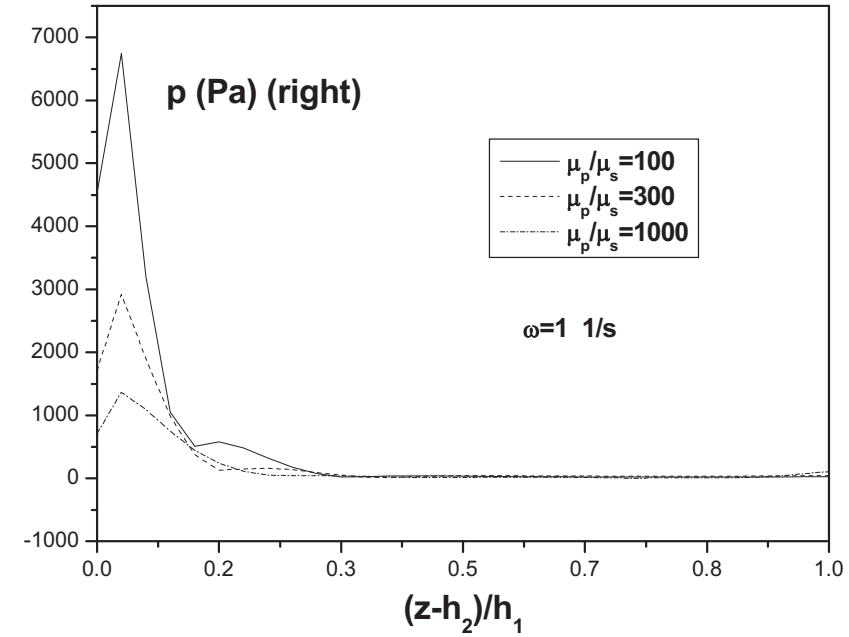

Fig. (3d). The influence of the modulus ratio between the pile and the seabed ( $\left.\mu_{p} / \mu_{s}, \lambda_{p} / \lambda_{s}\right)$ on the pore pressure of the seabed contacting the right side of the pile ( $p_{\text {right }}$ ) for the case of $\omega=1$ $[1 / \mathrm{s}]$.

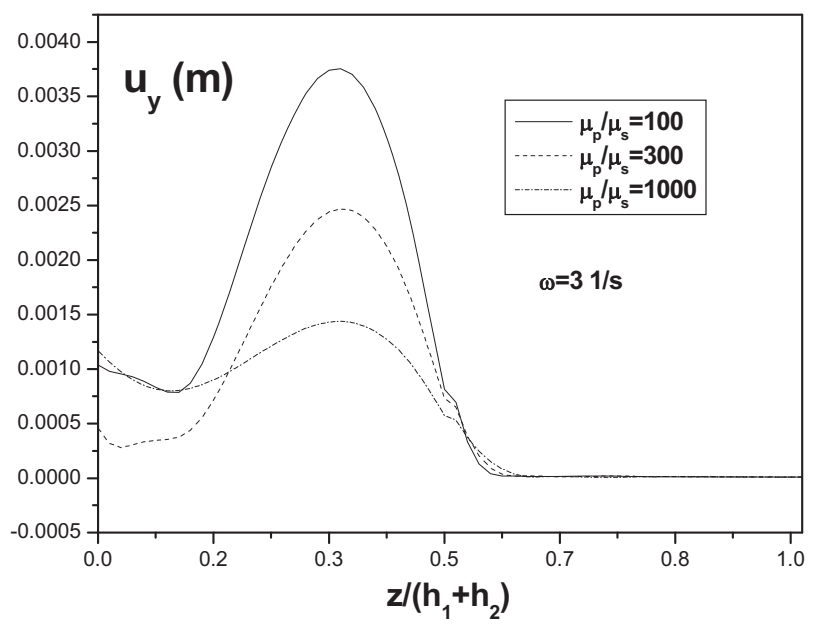

Fig. (4a). The influence of the modulus ratio between the pile and the seabed $\left(\mu_{p} / \mu_{s}, \lambda_{p} / \lambda_{s}\right)$ on the horizontal displacement $\left(u_{y}\right)$ for the case of $\omega=3[1 / \mathrm{s}]$.

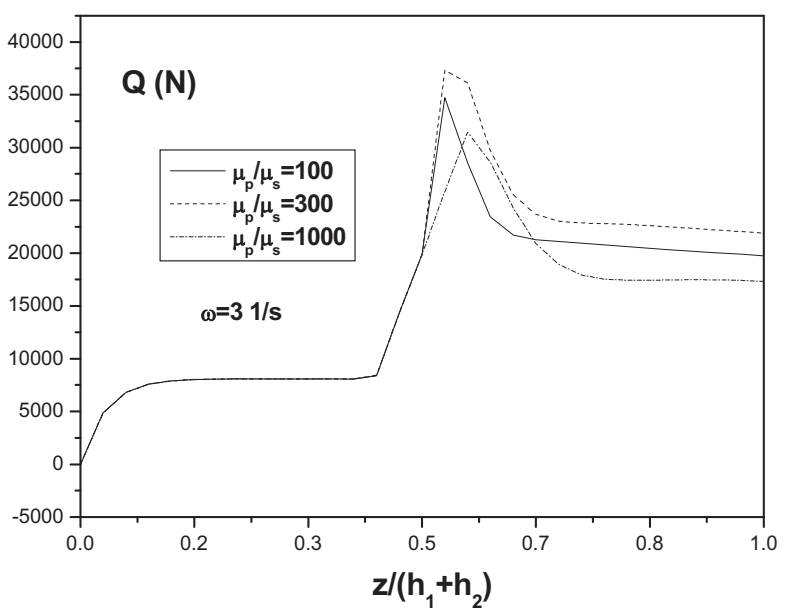

Fig. (4b). The influence of the modulus ratio between the pile and the seabed $\left(\mu_{p} / \mu_{s}, \lambda_{p} / \lambda_{s}\right)$ on the shear force of the pile (Q) for the case of $\omega=3[1 / \mathrm{s}]$.

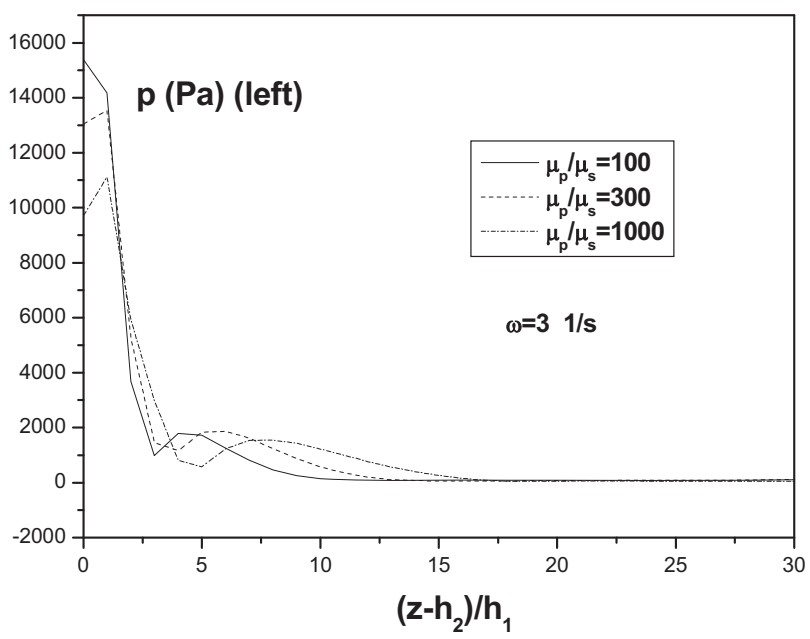

Fig. (4c). The influence of the modulus ratio between the pile and the seabed $\left(\mu_{p} / \mu_{s}, \lambda_{p} / \lambda_{s}\right)$ on the pore pressure of the seabed contacting the left side of the pile ( $p_{\text {left }}$ ) for the case of $\omega=3$ $[1 / \mathrm{s}]$.

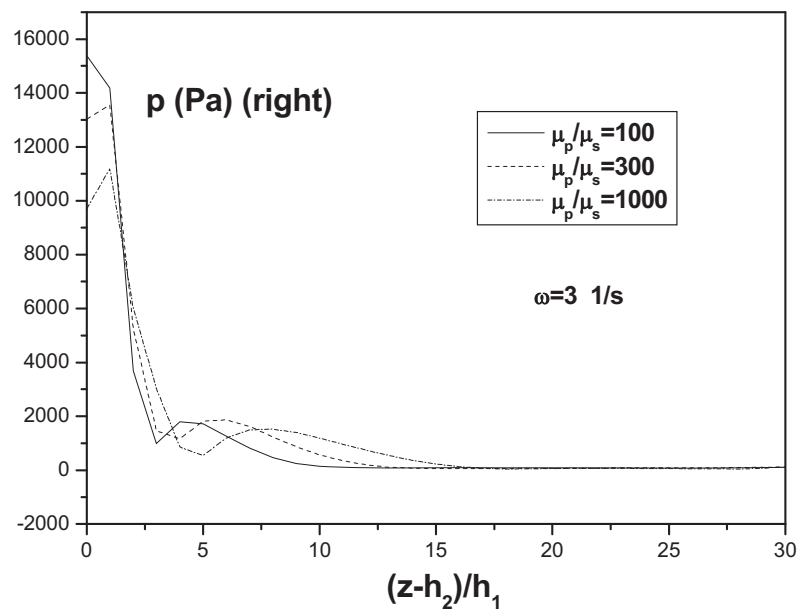

Fig. (4d). The influence of the modulus ratio between the pile and the seabed $\left(\mu_{p} / \mu_{s}, \lambda_{p} / \lambda_{s}\right)$ on the pore pressure of the seabed contacting the right side of the pile ( $p_{\text {right }}$ ) for the case of $\omega=3$ $[1 / \mathrm{s}]$. 


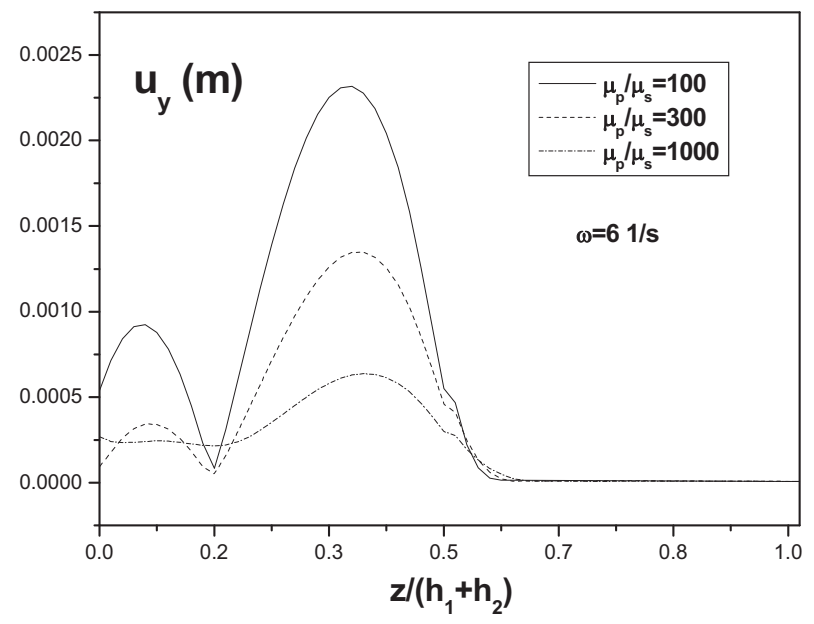

Fig. (5a). The influence of the modulus ratio between the pile and the seabed $\left(\mu_{p} / \mu_{s}, \lambda_{p} / \lambda_{s}\right)$ on the horizontal displacement $\left(u_{y}\right)$ for the case of $\omega=6[1 / \mathrm{s}]$.

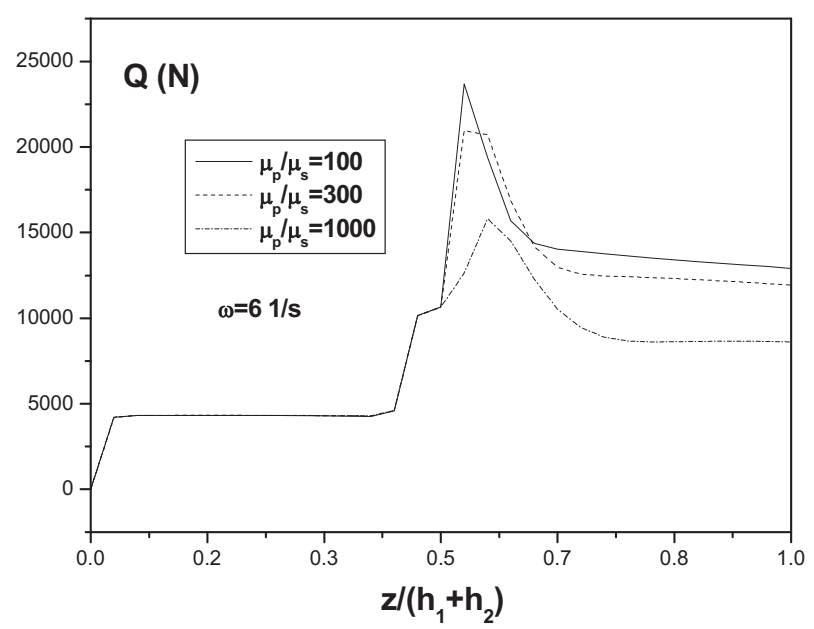

Fig. (5b). The influence of the modulus ratio between the pile and the seabed ( $\left.\mu_{p} / \mu_{s}, \lambda_{p} / \lambda_{s}\right)$ on the shear force of the pile (Q) for the case of $\omega=6[1 / \mathrm{s}]$.

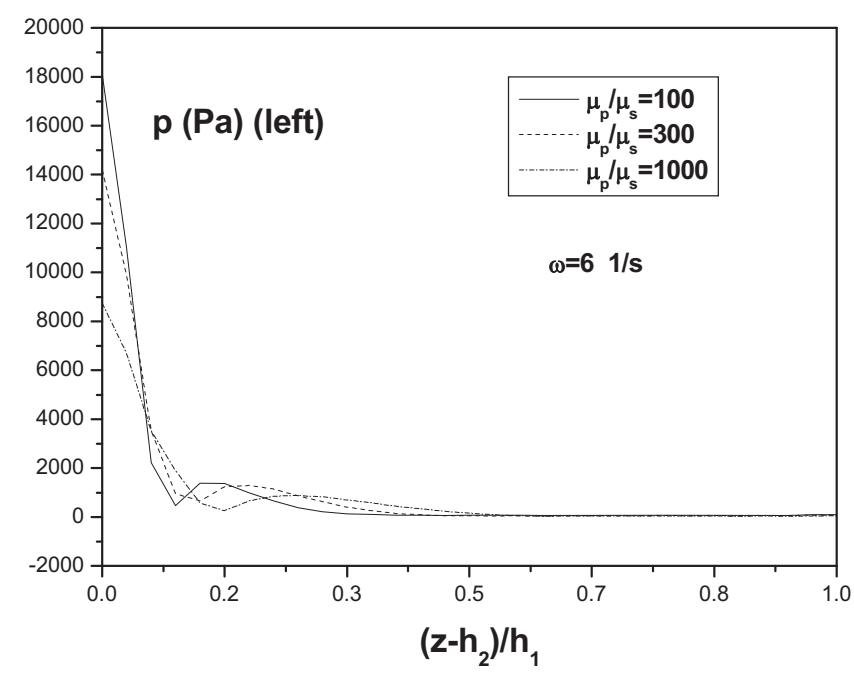

Fig. (5c). The influence of the modulus ratio between the pile and the seabed $\left(\mu_{p} / \mu_{s}, \lambda_{p} / \lambda_{s}\right)$ on the pore pressure of the seabed contacting the left side of the pile ( $p_{\text {left }}$ ) for the case of $\omega=6$ $[1 / \mathrm{s}]$.

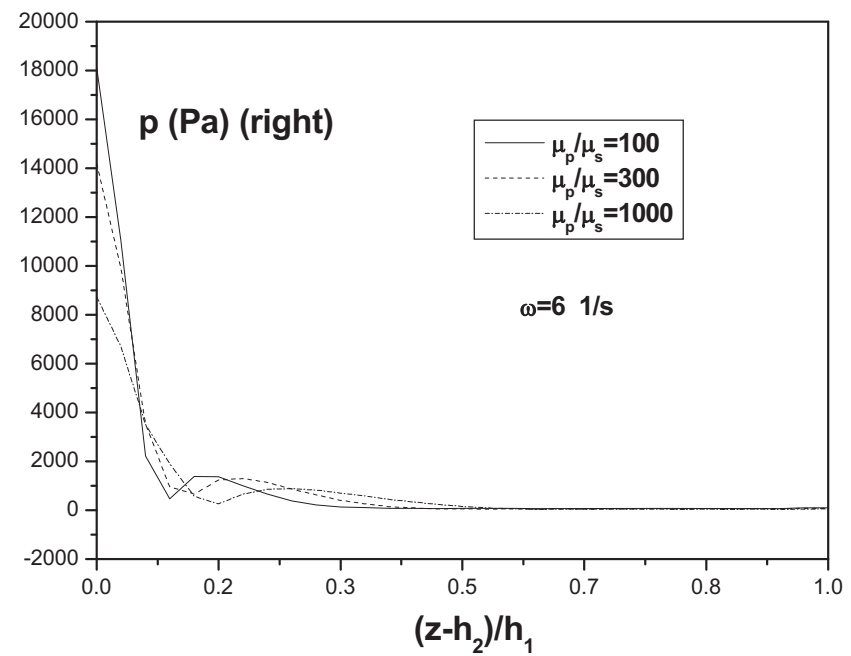

Fig. (5d). The influence of the modulus ratio between the pile and the seabed $\left(\mu_{p} / \mu_{s}, \lambda_{p} / \lambda_{s}\right)$ on the pore pressure of the seabed contacting the right side of the pile ( $p_{\text {right }}$ ) for the case of $\omega=6$ $[1 / \mathrm{s}]$.

Similarly, the shear force of the pile also increases when the angular frequencies of the water wave increases from $\omega=1$ to $\omega=3$, while it decreases when the angular frequency varies from $\omega=3$ to $\omega=6$. For the shear force for the part of the pile immersed in the seawater, the influence of the modulus ratio between the pile and the seabed is very little. For the shear force of the pile part embedded in the seabed, for the case of $\omega=1$, it increases markedly when the modulus ratio varies from $\mu_{p} / \mu_{s}=100$ to $\mu_{p} / \mu_{s}=1000$.

However, for the case of $\omega=3$, when the modulus ratio varies from $\mu_{p} / \mu_{s}=100$ to $\mu_{p} / \mu_{s}=300$, the shear force of the embedded part increases, while it decreases when the modulus ratio varies from $\mu_{p} / \mu_{s}=300$ to $\mu_{p} / \mu_{s}=1000$.

For the case of $\omega=6$, the shear force decreases as the modulus ratio increases.

For the pore pressure, we see that for all three cases of $\omega=1,3,6$, the pore pressure of the seabed decreases as the modulus ratio increases. Also, the maximum pore pressure usually occurs at the upper part of the seabed, which suggests that when performing the pile foundation design, the upper part of the seabed around the pile needs special treatment to improve its resistance against the liquefaction and shear failure. Numerical results concerning the pore pressure also illustrate that the pore pressure of the seabed at the left and the right side of the pile has very little difference in it amplitudes. Obviously, this phenomenon is due to the low frequency of the incident water wave, which suppresses the shelter effect of the pile.

Fig. (6) suggests that the pore pressures of the seabed at the left and the right side of the pile have phase differences. Thus, it can be concluded that the pore pressures of the left and the right side seabed don't achieve their peaks simultaneously. Note that to have a clear image, only the real part and the imaginary part of the pore pressure for the case of $\mu_{p} / \mu_{s}=100$ are plotted in Fig. (6). 


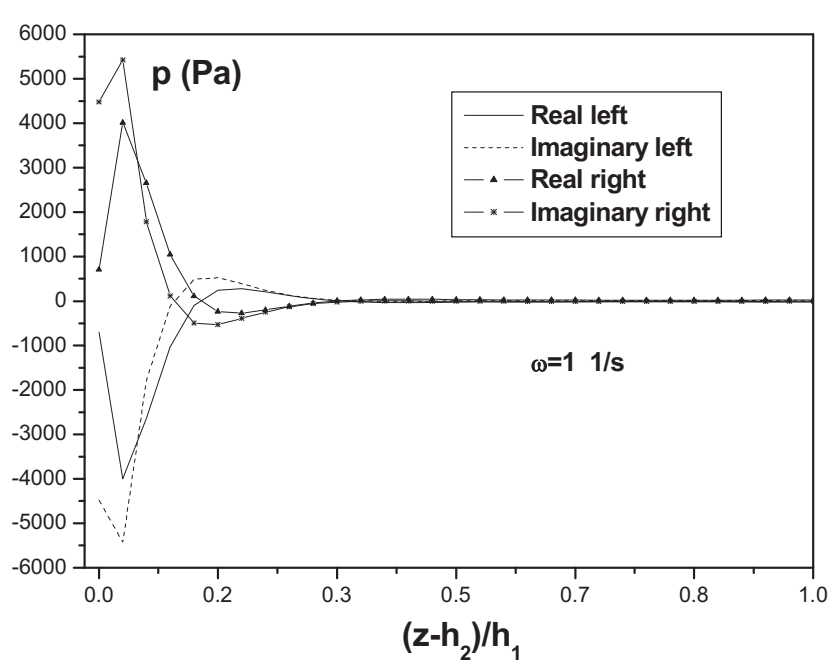

Fig. (6a). The real part and the imaginary part of the pore pressure of the seabed contacting the left and the right side of the pile for the case of $\mu_{p} / \mu_{s}=100, \lambda_{p} / \lambda_{s}=100$ with $\omega=1[1 / \mathrm{s}]$.

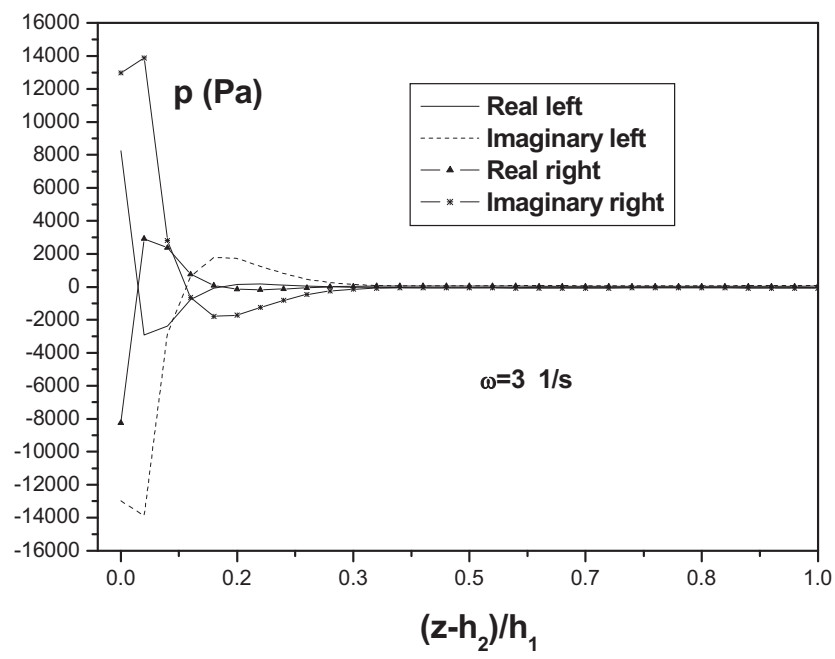

Fig. (6b). The real part and the imaginary part of the pore pressure of the seabed contacting the left and the right side of the pile for the case of $\mu_{p} / \mu_{s}=100, \lambda_{p} / \lambda_{s}=100$ with $\omega=3[1 / \mathrm{s}]$.

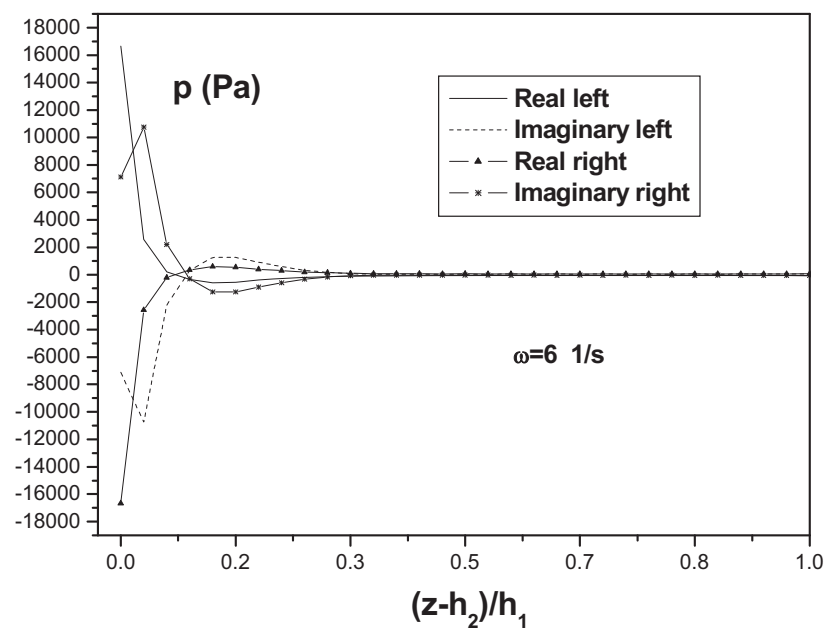

Fig. (6c). The real part and the imaginary part of the pore pressure of the seabed contacting the left and the right side of the pile for the case of $\mu_{p} / \mu_{s}=100, \lambda_{p} / \lambda_{s}=100$ with $\omega=6[1 / \mathrm{s}]$.

\section{CONCLUSIONS}

Based on the boundary element methods for the poroelastic medium and the acoustic medium, a frequency domain coupled model, which can account for the dynamic interaction between the pile, the seabed and the seawater when the system is subjected to linear water waves, was developed in this study. Although only dynamic response of the pile-seabed-seawater system to linear water waves is considered, the proposed model is general enough to be used to address other dynamic problems related to the coupled system. For example, using the Fourier transform method, the response of the coupled system to random waves in the time domain can be dealt with by the model. Furthermore, our model can be used to evaluate the dynamic response of the system to nonlinear water waves, seismic waves, wind loads or ice loads. To show the capacity of the proposed model, one numerical example is presented in the paper. Numerical results of the example indicate that maximum pore pressure usually occurs at the upper part of the seabed around the pile. Consequently, it is important to perform the design for the offshore pile foundation appropriately to lower the pore pressure at that region. Also, our calculation suggests that increasing modulus ratio between the pile and the seabed is an effective approach for reducing the maximum pore pressure of the seabed. Moreover, it can reduce the horizontal displacement of the pile considerably.

\section{ACKNOWLEDGEMENT}

This project is supported by the National Natural Science Foundation of China with grant number No. 50578071. The first author would also like to acknowledge the financial support received from the Chinese Education Department for returned oversea scholars.

\section{REFERENCES}

[1] W. Smith and A. D. Gordon, "Large breakwater toe failures", Journal of Waterways, Harbor and Coastal Engineering Division ASCE, vol. 109: pp. 253-255, 1983.

[2] H. Lundgren, J. H. C. Lindhardt and C. J. Romhild, "Stability of breakwaters on porous foundations", Proceedings of the 12th International Conference on Soil Mechanics and Foundation Engineering, pp. 451-454, 1989.

[3] R. Silvester and J. R. C. Hsu, "Sines revisited", Journal of Waterways, Port, Coastal and Ocean Engineering ASCE, vol. 115, pp. 327-344, 1989

[4] J. R. Morison, M. P. O’Brien, J. W. Johnson and S. A. Schaaf, "The forces exerted by surface waves on piles.", Journal of Petroleum Technology, Petroleum Transactions, AIME, vol. 189, pp. 149-154, 1950.

[5] R. C. MacCamy and R. A. Fuchs, "Waves forces on piles: a diffraction theory", U.S. Army Corps of Engineering, Beach Erosion Board, Washington, D.C., technical Memorandum No. 69, 1954.

[6] S. K. Chakarabarti and A. Tam, "Interaction of waves with large vertical cylinder”, Journal of Ship Research, vol. 19: 22-23, 1975.

[7] W. H. Tang, "Uncertainties in offshore axial pile capacity", In: (F.H. Kulhawy, Ed.). Foundation Engineering: Current Principles and Practices. ASCE Press, New York, pp. 833-847, 1989.

[8] J. A. Eicher, H. Guan, D.-S. Jeng, "Stress and deformation of offshore piles under structural and wave loading", Ocean Engineering, vol. 30, pp. 369-385, 2003.

[9] H. Mitwally and M. Novak, "Response of offshore towers with pile interaction", Journal of Engineering Mechanics ASCE, vol. 113, pp. 1065-1084, 1987.

[10] Y. E. Mostafa and M. H. E. Naggar, "Response of fixed offshore platforms to wave and current loading including soil-structure interaction", Soil Dynamics and Earthquake Engineering vol. 24, pp. 357-368, 2004. 
[11] C. Mei, The applied dynamics of Ocean surface waves. John Wiley \& Sons Inc., 1983.

[12] M. A. Biot, "Theory of propagation of elastic waves in a fluidsaturated porous solid, I, Low frequency range", J. Acoust. Soc. Am., vol. 28, pp. 168-178, 1956.

[13] M. A. Biot, "Mechanics of deformation and acoustic propagation in porous media", J. Appl. Phys. vol. 33, pp. 1482-1498, 1962.

[14] M. A. Biot, "General theory of three-dimensional consolidation", $J$. Appl. Phys., vol. 12, pp. 155-164., 1941.

[15] I. N. Sneddon, Fourier transforms, New York, NY: McGraw-Hill, 1951.

[16] G. Bonnet, "Basic singular solutions for a poroelastic medium in the dynamic range", J. Acoust. Soc. Am., vol. 82, pp. 1758-1762, 1982.

[17] J. Dominguez, "An integral formulation for dynamic poroelasticity", J. Appl. Mech., ASME, vol. 58, pp. 588-591, 1991.

[18] J. Dominguez, "Boundary element approach for dynamic poroelastic problems", Int. J. Numer. Methods Eng. vol. 35, pp. 307-324, 1992.

[19] A. H. D. Cheng, D. E. Badmus, D E. Beskos, "Integral equations for dynamic poroelasticity in frequency domain with BEM solution", J. Eng. Mech. ASCE, vol. 117, pp. 1136-1157, 1991.
[20] C. Zimmerman and M. Stern, "Boundary element solution of 3-D wave scatter problems in a poroelastic medium", Eng. Anal. Bound. Elem. vol. 12, pp. 223-240, 1993.

[21] A. N. Norris, "Radiation from a point source and scattering theory in a fluid-saturated porous medium", J. Acoust. Soc. Am., vol. 77, pp. 2012-2022, 1985.

[22] J. F. Lu and D.S. Jeng, "A half-space saturated poroelastic medium subject to a moving point load", International Journal of Solid and Structures, vol. 44, pp. 573-586, 2007.

[23] A. F. Seybert and T. W. Wu, "Modified Helmholtz integral equation for bodies sitting on an infinite plane", J. Acoust. Soc. Am., vol. 85, 19-23, 1989

[24] R. D. Ciskowski and C. A. Brebbia, Boundary Element Methods in Acoustics. Springer, 1991.

[25] H. Deresiewicz and R. Skalak, "On uniqueness in dynamic poroelasticity", Bulletin of the Seismological Society of America, vol. 53, pp. 783-788, 1963.

[26] R. D. Stoll and T. K. Kan, "Reflection of acoustic waves at a watersediment interface", J. Acoust. Soc. Am., vol. 70, pp. 149-156, 1981.

(C) Lu and Jeng; Licensee Bentham Open.

This is an open access article distributed under the terms of the Creative Commons Attribution License (http://creativecommons.org/licenses/by/2.5/), which permits unrestrictive use, distribution, and reproduction in any medium, provided the original work is properly cited. 